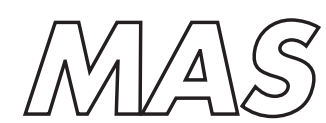

Modelling, Analysis and Simulation

\footnotetext{
Modelling, Analysis and Simulation

MAS Blooming in a non-local, coupled phytoplankton-nutrient model
}
A. Zagaris, A. Doelman, N.N. Pham Thi, B.P. Sommeijer
RePORT MAS-E0708 JUNe 2007 
Centrum voor Wiskunde en Informatica (CWI) is the national research institute for Mathematics and Computer Science. It is sponsored by the Netherlands Organisation for Scientific Research (NWO).

CWI is a founding member of ERCIM, the European Research Consortium for Informatics and Mathematics.

CWI's research has a theme-oriented structure and is grouped into four clusters. Listed below are the names of the clusters and in parentheses their acronyms.

Probability, Networks and Algorithms (PNA)

Software Engineering (SEN)

\section{Modelling, Analysis and Simulation (MAS)}

Information Systems (INS)

Copyright (C) 2007, Stichting Centrum voor Wiskunde en Informatica

P.O. Box 94079, 1090 GB Amsterdam (NL)

Kruislaan 413, 1098 SJ Amsterdam (NL)

Telephone +31205929333

Telefax +31205924199

ISSN 1386-3703 


\title{
Blooming in a non-local, coupled phytoplankton-nutrient model
}

\begin{abstract}
Recently, it has been discovered that the dynamics of phytoplankton concentrations in an ocean exhibit a rich variety of patterns, ranging from trivial states to oscillating and even chaotic behavior [J. Huisman, N.N. Pham Thi, D.M. Karl, and B.P. Sommeijer (2006), Reduced mixing generates oscillations and chaos in the oceanic deep chlorophyll maximum, Nature $439322-$ 325]. This paper is a first step towards understanding the bifurcational structure associated to non-local, coupled phytoplankton-nutrient models as studied in that paper. Its main subject is the linear stability analysis that governs the occurrence of the first nontrivial stationary patterns, the 'deep chlorophyll maxima' (DCMs) and the 'benthic layers' (BLs). Since the model can be scaled into a system with a natural singularly perturbed nature, and since the associated eigenvalue problem decouples into a problem of Sturm-Liouville type, it is possible to obtain explicit (and rigorous) bounds on, and accurate approximations of, the eigenvalues. The analysis yields bifurcation-manifolds in parameter space, of which the existence, position and nature are confirmed by numerical simulations. Moreover, it follows from the simulations and the results on the eigenvalue problem that the asymptotic linear analysis may also serve as a foundation for the secondary bifurcations, such as the oscillating DCMs, exhibited by the model.
\end{abstract}

2000 Mathematics Subject Classification: 35B20, 35B32, 34B24, 34E20, 86A05, 92D40.

Keywords and Phrases: Phytoplankton; Singular perturbations; Eigenvalue analysis; Sturm-Liouville; Airy functions; WKB.

Note: The work was carried out under subthemes MAS1.2 - Nonlinear Dynamics of Natural Systems - and MAS1.1 Scientific Computing in the Life Sciences. 



\title{
BLOOMING IN A NON-LOCAL, COUPLED PHYTOPLANKTON-NUTRIENT MODEL *
}

\author{
A. ZAGARIS $^{\dagger}$, A. DOELMAN ${ }^{\ddagger}$, N. N. PHAM THI ${ }^{\S}$, AND B.P. SOMMEIJER
}

\begin{abstract}
Recently, it has been discovered that the dynamics of phytoplankton concentrations in an ocean exhibit a rich variety of patterns, ranging from trivial states to oscillating and even chaotic behavior [J. Huisman, N. N. Pham Thi, D. M. Karl, and B. P. Sommeijer (2006), Reduced mixing generates oscillations and chaos in the oceanic deep chlorophyll maximum, Nature 439 322-325]. This paper is a first step towards understanding the bifurcational structure associated to non-local, coupled phytoplankton-nutrient models as studied in that paper. Its main subject is the linear stability analysis that governs the occurrence of the first nontrivial stationary patterns, the 'deep chlorophyll maxima' (DCMs) and the 'benthic layers' (BLs). Since the model can be scaled into a system with a natural singularly perturbed nature, and since the associated eigenvalue problem decouples into a problem of Sturm-Liouville type, it is possible to obtain explicit (and rigorous) bounds on, and accurate approximations of, the eigenvalues. The analysis yields bifurcation-manifolds in parameter space, of which the existence, position and nature are confirmed by numerical simulations. Moreover, it follows from the simulations and the results on the eigenvalue problem that the asymptotic linear analysis may also serve as a foundation for the secondary bifurcations, such as the oscillating DCMs, exhibited by the model.
\end{abstract}

Key words. phytoplankton, singular perturbations, eigenvalue analysis, Sturm-Liouville, Airy functions, WKB.

AMS subject classifications. 35B20, 35B32, 34B24, 34E20, 86A05, 92D40.

1. Introduction . Phytoplankton forms the foundation of most aquatic ecosystems [16]. Since it transports significant amounts of atmospheric carbon dioxide into the deep oceans, it may play a crucial role in climate dynamics [6]. Therefore, the dynamics of phytoplankton concentrations have been studied intensely and from various points of view (see, for instance, $[7,11,15]$ and the references therein). Especially relevant and interesting patterns exhibited by phytoplankton are the 'deep chlorophyll maxima' (DCMs), or phytoplankton blooms, in which the phytoplankton concentration exhibits a maximum at a certain, well-defined depth of the ocean (or, in general, of a vertical water column). Simple, one-dimensional, scalar-but non-local-models for the influence of a depth-dependent light intensity on phytoplankton blooms have been studied since the early eighties already [14]. The non-locality of these models is a consequence of the influence of the accumulated plankton concentration on the light intensity at a certain depth $z$ (see (1.2) below). Numerical simulations and various mathematical approaches (see $[5,7,8,10,12]$ ) show that these models may, indeed, exhibit DCMs, depending on the manner in which the decay of the light intensity with depth is modelled and for certain parameter combinations.

The analysis in [14] establishes that, for a certain (large) class of light intensity functions, the scalar model has a stationary global attractor. This attractor may be trivial, i.e., the phytoplankton concentration $W$ may decrease in time to $W \equiv 0$. If this trivial pattern is spectrally unstable, either the global attractor is a DCM or the phytoplankton concentration is maximal at the surface of

*This work was supported by the Netherlands Organisation for Scientific Research (NWO).

$\dagger$ University of Amsterdam, Korteweg-de Vries Institute, Plantage Muidergracht 24, 1018 TV Amsterdam, the Netherlands, and Centrum voor Wiskunde en Informatica (CWI), P.O. Box 94079, 1090 GB Amsterdam, the Netherlands (A.Zagaris@cwi.nl)

$¥$ CWI, P.O. Box 94079, 1090 GB Amsterdam, the Netherlands, and University of Amsterdam, Korteweg-de Vries Institute, Plantage Muidergracht 24, 1018 TV Amsterdam, the Netherlands (A.Doelman@cwi.nl)

$\S$ ABN AMRO Bank N.V., P.O. Box 283, 1000 EA, Amsterdam, the Netherlands (Nga.Pham.Thi@nl.abnamro.com)

ฯ CWI, P.O. Box 94079, 1090 GB Amsterdam, the Netherlands (B.P.Sommeijer@cwi.nl) 
the ocean (this latter case is called a 'surface layer' (SL) [10, 15]). It should be noted here that 'benthic layers' (BLs) [15]-i.e., phytoplankton blooms that become maximum at the bottom of the water column - cannot occur in the setting of [14], due to the choice of boundary conditions. Although the analysis in [14] cannot be applied directly to all scalar models in the literature, the main conclusion - that such models may only exhibit stationary nontrivial patterns (DCMs, SLs, or BLs) — seems to be true for each one of these models.

In sharp contrast to this, it has been numerically discovered recently [11] that systems-i.e., non-scalar models in which the phytoplankton concentration $W$ is coupled to an equation for a nutrient $N$-may exhibit complex behavior ranging from periodically oscillating DCMs to chaotic dynamics. These non-stationary DCMs have also been observed in the Pacific Ocean [11].

In this paper, a first step towards understanding the rich dynamics of the phytoplankton models considered in [11] is taken. Following [11], we consider the one-dimensional (i.e., depth-dependent only), non-local model,

$$
\left\{\begin{array}{l}
W_{t}=D W_{z z}-V W_{z}+[\mu P(L, N)-l] W \\
N_{t}=D N_{z z}-\alpha \mu P(L, N) W
\end{array}\right.
$$

for $(z, t) \in\left[0, z_{B}\right] \times \mathbf{R}_{+}$and where $z_{B}>0$ determines the depth of the water column. The system is assumed to be in the turbulent mixing regime (see, for instance, $[5,10]$ ), and thus the diffusion coefficient $D$ is taken to be identically the same for $W$ and $N$. The parameters $V, l, \alpha$ and $\mu$ measure, respectively, the sinking speed of phytoplankton, the species-specific loss rate, the conversion factor and the maximum specific production rate, and they are all assumed to be positive (see Remark 1.1 also). The light intensity $L$ is modeled by

$$
L(z, t)=L_{I} \mathrm{e}^{-K_{b g} z-R \int_{0}^{z} W(\zeta, t) d \zeta},
$$

where $L_{I}$ is the intensity of the incident light at the water surface, $K_{b g}$ is the light absorption coefficient due to non-plankton components and $R$ is the light absorption coefficient due to the plankton. Note that $L$ introduces the non-locality in the system. The function $P(L, N)$, which is responsible for the coupling, models the influence of light and nutrient on the phytoplankton growth and is taken to be

$$
P(L, N)=\frac{L N}{\left(L+L_{H}\right)\left(N+N_{H}\right)},
$$

where $L_{H}$ and $N_{H}$ are the half-saturation constants of light and nutrient, respectively. We note that, from a qualitative standpoint, the particular form of $P$ is of little importance. Different choices for $P$ yield the same qualitative results, as long as they share certain common characteristics with the function given in (1.3), see Remark 1.1. Finally, we equip the system with the boundary conditions

$$
D W_{z}-\left.V W\right|_{z=0, z_{B}}=0,\left.\quad N_{z}\right|_{z=0}=0 \quad \text { and }\left.\quad N\right|_{z=z_{B}}=N_{B}
$$

i.e., no-flux through the boundaries except at the bottom of the column where $N$ is at its maximum (prescribed by $N_{B}$ ). We refer to Remark 1.1 for a discussion of more general models. To recast the model in nondimensional variables, we rescale time and space by setting

$$
x=z / z_{B} \in(0,1) \quad \text { and } \quad \tau=\mu t \geq 0,
$$

introduce the scaled phytoplankton concentration $\omega$, nutrient concentration $\eta$ and light intensity $j$,

$$
\omega(x, \tau)=\frac{l \alpha z_{B}^{2}}{D N_{B}} W(z, t), \quad \eta(x, \tau)=\frac{N(z, t)}{N_{B}}, \quad j(x, \tau)=\frac{L(z, t)}{L_{I}}
$$


and thus recast (1.1) in the form

$$
\left\{\begin{array}{l}
\omega_{\tau}=\varepsilon \omega_{x x}-\sqrt{\varepsilon} a \omega_{x}+(p(j, \eta)-\ell) \omega \\
\eta_{\tau}=\varepsilon\left(\eta_{x x}-\frac{1}{\ell} p(j, \eta) \omega\right)
\end{array}\right.
$$

Here,

$$
j(x, \tau)=\exp \left(-\kappa x-r \int_{0}^{x} \omega(\chi, \tau) d \chi\right), \quad \text { with } \quad \kappa=K_{b g} z_{B} \quad \text { and } \quad r=\frac{R D N_{B}}{l \alpha z_{B}}
$$

and

$$
\varepsilon=\frac{D}{\mu z_{B}^{2}}, \quad a=\frac{V}{\sqrt{\mu D}}, \quad \ell=\frac{l}{\mu} \quad \text { and } \quad p(j, \eta)=\frac{j \eta}{\left(j+j_{H}\right)\left(\eta+\eta_{H}\right)},
$$

where $j_{H}=L_{H} / L_{I}, \eta_{H}=N_{H} / N_{B}$. The rescaled boundary conditions are given by

$$
\left(\sqrt{\varepsilon} \omega_{x}-a \omega\right)(0)=\left(\sqrt{\varepsilon} \omega_{x}-a \omega\right)(1)=0, \quad \eta_{x}(0)=0 \quad \text { and } \quad \eta(1)=1 .
$$

These scalings are suggested by realistic parameter values in the original model (1.1) as reported in [11]. Typically,

$$
D \approx 0.1 \mathrm{~cm}^{2} / \mathrm{s}, \quad V \approx 4.2 \mathrm{~cm} / \mathrm{h}, \quad z_{B} \approx 3 \cdot 10^{4} \mathrm{~cm}, \quad l \approx 0.01 / \mathrm{h} \quad \text { and } \quad \mu \approx 0.04 / h,
$$

so that

$$
\varepsilon \approx 10^{-5}, \quad a \approx 1 \text { and } \ell \approx 0.25
$$

in (1.5). Thus, realistic choices of the parameters in (1.1) induce a natural singularly perturbed structure in the model, as is made explicit by the scaling of (1.1) into (1.5). In this article, $\varepsilon$ shall be considered as an asymptotically small parameter, i.e., $0<\varepsilon \ll 1$.

The simulations in [11] indicate that the DCMs bifurcate from the trivial stationary pattern,

$$
\bar{\omega}(x, \tau) \equiv 0, \quad \bar{\eta}(x, \tau) \equiv 1, \quad \text { for all } \quad(x, \tau) \in[0,1] \times \mathbf{R}_{+},
$$

see also Section 7. To analyze this (first) bifurcation, we set

$$
(\omega(x, \tau), \eta(x, \tau))=\left(\tilde{\omega} e^{\lambda \tau}, 1+\tilde{\eta} e^{\lambda \tau}\right), \quad \text { with } \quad \lambda \in \mathbf{C}
$$

and consider the (spectral) stability of $(\bar{\omega}, \bar{\eta})$. This yields the linear eigenvalue problem,

$$
\left\{\begin{aligned}
\varepsilon \omega_{x x}-\sqrt{\varepsilon} a \omega_{x}+(f(x)-\ell) \omega & =\lambda \omega \\
\varepsilon\left(\eta_{x x}-\frac{1}{\ell} f(x) \omega\right) & =\lambda \eta
\end{aligned}\right.
$$

where we have dropped the tildes with a slight abuse of notation. The linearized boundary conditions are given by (1.8) here also, while the function $f$ is the linearization of the function $p(j, \eta)$,

$$
f(x)=\frac{1}{\left(1+\eta_{H}\right)\left(1+j_{H} \mathrm{e}^{\kappa x}\right)} .
$$

The linearized system (1.11) is partially decoupled, so that the stability of $(\bar{\omega}, \bar{\eta})$ as solution of the two-component system (1.5) is determined by a one-component Sturm-Liouville problem,

$$
\begin{aligned}
\varepsilon \omega_{x x}-\sqrt{\varepsilon} a \omega_{x}+(f(x)-\ell) \omega & =\lambda \omega, \\
\left(\sqrt{\varepsilon} \omega_{x}-a \omega\right)(0)=\left(\sqrt{\varepsilon} \omega_{x}-a \omega\right)(1) & =0 .
\end{aligned}
$$


Hence, we have returned to a scalar system as studied in $[5,7,8,10,12,14,15]$. However, our viewpoint differs significantly from that of these studies. The simulations in [11] (and Section 7 of the present article) suggest that the destabilization of $(\bar{\omega}, \bar{\eta})$ into a DCM is merely the first in a series of bifurcations. In fact, Section 7 shows that this DCM undergoes 'almost immediately' a second bifurcation of Hopf type, i.e., it begins to oscillate periodically in time. According to [14], this is impossible in a scalar model (also, it has not been numerically observed in such models), and so the Hopf bifurcation must be induced by the weak coupling between $\omega$ and $\eta$ in the full model (1.5).

Our analysis establishes that the eigenvalue $\lambda_{0}$ of (1.13) that induces the (stationary) DCM as it crosses through zero is the first of a sequence of eigenvalues $\lambda_{n}$ that are only $\mathcal{O}\left(\varepsilon^{1 / 3}\right)$ apart (where $\varepsilon^{1 / 3} \approx 0.045$, see Fig. 7.3). The simulations in Section 7 show that the distance between this bifurcation and the subsequent Hopf bifurcation of the DCM is of the same magnitude (see Fig. 7.3 especially). Thus, the (stationary) DCM already destabilizes when $\lambda_{0}$ is still asymptotically small (in $\varepsilon$ ), which indicates that the amplitude of the bifurcating DCM is also still asymptotically small and determined (at leading order) by $\omega_{0}(x)$, the eigenfunction associated with $\lambda_{0}$. This agrees fully with our linear stability analysis, since $\omega_{0}(x)$ indeed has the structure of a DCM (see Sections 2 and 6). As a consequence, the leading order (in $\varepsilon$ ) stability analysis of the DCM is also governed by the partially decoupled system (1.11). In other words, although it is the coupling between $\omega(x)$ and $\eta(x)$ in (1.5) that drives the secondary bifurcation(s), the leading order analysis is governed by the eigenvalues and eigenfunctions of (1.13). Naturally, the next eigenvalues $\lambda_{1}, \lambda_{2}, \ldots$, and their associated eigenfunctions will play a key role in such a secondary bifurcation analysis.

Therefore, a detailed knowledge of the nature of the eigenvalues and eigenfunctions of (1.13) forms the foundation of analytical insight in the bifurcations exhibited by (1.5). This is the topic of the present paper; the subsequent (weakly) nonlinear analysis is the subject of work in progress.

The structure of the eigenvalue problem (1.13) is rather subtle, and therefore we employ two different analytical approaches. First, in Sections 3-5, we derive explicit and rigorous bounds on the eigenvalues in terms of expressions based on the zeroes of the Airy function of the first kind (and its derivative), see Theorem 2.1. This theorem establishes the existence of the aforementioned eigenvalue sequence which is associated to the bifurcation of the DCM and that these eigenvalues are $\mathcal{O}\left(\varepsilon^{1 / 3}\right)$ apart. There is, however, another eigenvalue one that is associated with the bifurcation of a BL. Although this eigenvalue also appears for biologically relevant parameter combinations in the unscaled model (1.1), this bifurcation has not been observed in [11]. This eigenvalue is isolated, in the sense that it is not part of the eigenvalue sequence associated with the DCMs-instead, it corresponds to a zero of a linear combination of the Airy function of the second kind and its derivative. Depending on the relative strength of the nondimensionless parameter $a$ and of the parameters appearing in (1.12), the trivial state $(\bar{\omega}, \bar{\eta})$ either bifurcates into a DCM or into a BL. Our analysis establishes the bifurcation sets explicitly in terms of the parameters in the problem and is numerically confirmed by simulations in Section 7. Note that the co-dimension 2 point, at which the DCM and BL bifurcate simultaneously and which we determine explicitly, is somewhat similar to that studied in [20]. Nevertheless, the differences are crucial. For instance, [20] considers a two-layer ODE model where, additionally, the DCM interacts with a SL instead of a BL (a SL cannot occur in our setting because $V>0$ in (1.1), see Remark 1.1). Our analysis of (1.13) is completed by a WKB approach in Section 6. Using this method, we deduce that the critical eigenfunctions have, indeed, the structures of a DCM or a BL. The outcome of our analysis is summarized in Section 2.

The paper is concluded by Section 7, where we compare our analytical results on the first bifurcations of (1.5) with numerical simulations of the full model. Although our insights are only based on linear predictions, and we do not yet have analytical results on the (nonlinear) stability of the patterns that bifurcate, we do find that there is an excellent agreement between the linear analysis and the numerical simulations. Thus, our analysis of (1.13) yields explicit bifurcation curves 
in the biological parameter space associated to (1.1). For any given values of the parameters, our analysis predicts whether one may expect a phytoplankton pattern with the structure of a (possibly oscillating) DCM, a pattern with the structure of a BL, or whether the phytoplankton will become extinct. Moreover, we also briefly consider secondary bifurcations into time-periodic patterns. These bifurcations are not directly covered by our linear analysis, but the distance between the first and second bifurcation in parameter space implies that the linearized system (1.13) must play a crucial role in the subsequent (weakly) nonlinear analysis, see the discussion above.

REMARK 1.1. Although we focus on model (1.1) (equivalently, (1.5)) in our analysis, our approach and our findings are also applicable and relevant for more extensive models:

- In [11], (1.1) was extended to a model for various phytoplankton species $W_{i}(z, t)(i=1, \ldots, n)$. A stability analysis of the trivial pattern $W_{i} \equiv 0, N \equiv N_{B}$ yields $n$ uncoupled copies of (1.13) in which the parameters depend on the species, i.e. on the index $i$. As a consequence, the results of this paper can also be applied to this multi-species setting.

- It is natural to include the possibility of horizontal flow and diffusion in the model (1.1). In the most simple setting, this can be done by allowing $W$ and $N$ to vary with $(x, y, z, t)$ and to include horizontal diffusion terms in (1.1), i.e., $D_{H}\left(W_{x x}+W_{y y}\right)$ and $D_{H}\left(N_{x x}+N_{y y}\right)$ with $D_{H} \neq D$, in general-see [17], for instance. Again, the linear stability analysis of the trivial state is essentially not influenced by this extension. The exponentials in the Ansatz following (1.10) now need to be replaced by $\exp (\lambda \tau+i(k \tilde{x}+l \tilde{y}))$, where $k$ and $l$ are wave numbers in the (rescaled) $x$ and $y$ directions. As a consequence, one only has to replace $\ell$ by $\ell-D_{H}\left(k^{2}+l^{2}\right)$ in (1.13).

- Neither the character, nor the fact that we assign specific formulas to the growth and light intensity functions $P(L, N)$ (see (1.3)) and $L(z, t)$ (see (1.2)) is essential for our analysis. One only needs that $f(x)$ is decreasing and bounded in $[0,1]$ - both assumptions are natural from a biological standpoint.

- We have considered 'sinking' phytoplankton species in our model, i.e., $V>0$ in (1.1) and thus $a>0$ in (1.13). Our analysis can also be applied to buoyant species $(V \leq 0)$. In that case, the bifurcating DCMs may transform into SLs—-see, also, [10, 15].

- The values of $\varepsilon, a$ and $\ell$ in (1.9) are typical of oceanic settings [11]. These values differ in an estuary, and $\varepsilon$ can no longer be assumed to be asymptotically small, see [18] and the references therein. Moreover, phytoplankton blooms in an estuary are strongly influenced by the concentration of suspended sediment and typically occur not only at a certain depth $z$, but also at a certain horizontal position in the estuary. Thus, (1.13) must be extended to account for such blooms; however, it may still play an important role as a limiting case or a benchmark [18].

2. The main results . First, we define the function $F$ through

$$
F(x)=F\left(x ; j_{H}, \kappa, \eta_{H}\right)=f(0)-f(x) \geq 0, \quad \text { for all } \quad x \in[0,1],
$$

see $(1.12)$, and the constants $\sigma_{L}=\sigma_{L}\left(\kappa, j_{H}, \eta_{H}\right)$ and $\sigma_{U}=\sigma_{U}\left(\kappa, j_{H}, \eta_{H}\right)$ so that

$$
\sigma_{L} x \leq F(x) \leq \sigma_{U} x, \quad \text { for all } x \in[0,1]
$$

Note that the optimal values of $\sigma_{U}$ and $\sigma_{L}$ can be determined explicitly. This (simple but technical) analysis is postponed until after the formulation of Theorem 2.1, see Lemma 2.1 and Figures 2.2 and 2.3. Furthermore, we write $\mathrm{Ai}$ and $\mathrm{Bi}$ for the Airy functions of the first and second kind [1], respectively, and $A_{n}<0$ for the $n$-th zero of $\mathrm{Ai}(x)$, see Fig. 2.1. We also define the functions

$$
\Gamma(\mathrm{Ai}, x)=\operatorname{Ai}(x)-2 \varepsilon^{1 / 6} \sigma^{1 / 3} a^{-1} \mathrm{Ai}^{\prime}(x), \quad \Gamma(\mathrm{Bi}, x)=\operatorname{Bi}(x)-2 \varepsilon^{1 / 6} \sigma^{1 / 3} a^{-1} \operatorname{Bi}^{\prime}(x),
$$

(see Fig. 2.1 and Section 4.1), with $a$ as in (1.7) and $\sigma$ an a priori parameter (later, it will be set to either $\sigma_{L}$ or $\left.\sigma_{U}\right)$. We let $A_{n, \sigma}^{\prime}, n=1,2, \ldots$, be the $n$-th zero of $\Gamma(\mathrm{Ai}, x)$ and $B_{0, \sigma}$ be the positive zero of $\Gamma\left(\mathrm{Bi}, \varepsilon^{-1 / 3} \sigma^{1 / 3}(1+x)\right)$. Note that $A_{n, \sigma}^{\prime}$ is $\mathcal{O}\left(\varepsilon^{1 / 6}\right)$ close to $A_{n}$ and that $B_{0, \sigma}=a^{2} / 4 \sigma-1$ 

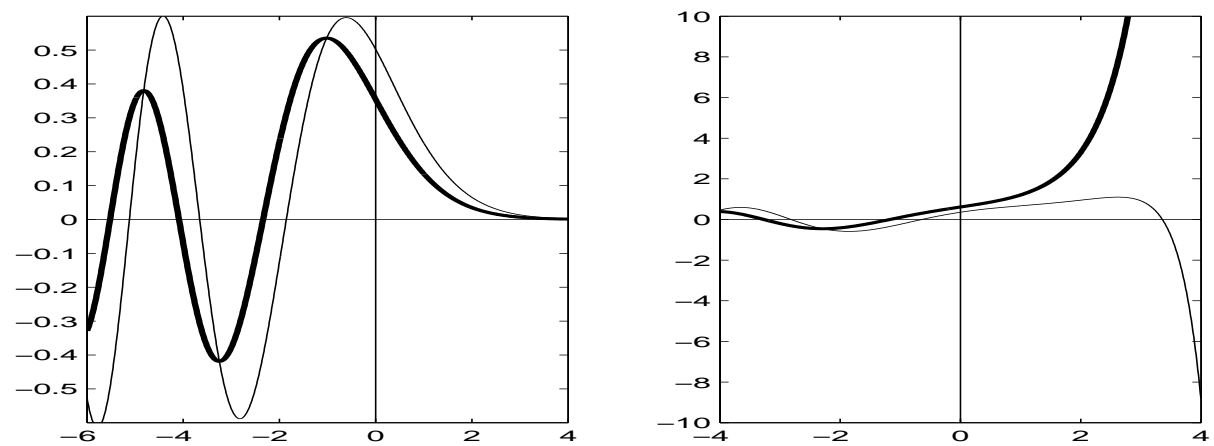

FIG. 2.1. The Airy functions of first and second kind (plotted in thick lines in the left and right panel, respectively) together with the functions $\Gamma(\mathrm{Ai}, x)$ and $\Gamma(\mathrm{Bi}, x)$ (plotted in thin lines). Here, $\varepsilon=0.1, a=3, \sigma=2$.

at leading order in $\varepsilon$ (see Lemma A.2 for more accurate estimates). Finally, we let (2.4) $\lambda^{*}=f(0)-\ell-a^{2} / 4, \quad \lambda_{0}^{*, \sigma}=\lambda^{*}+\sigma B_{0, \sigma} \quad$ and $\quad \lambda_{n}^{*, \sigma}=\lambda^{*}-\varepsilon^{1 / 3} \sigma^{2 / 3}\left|A_{n, \sigma}^{\prime}\right|, \quad n \in \mathbf{N}$, and we note that $\lambda_{0}^{*, \sigma}$ and $\lambda_{n}^{*, \sigma}$ are decreasing functions of $\sigma$. We can now formulate our main result.

TheOREM 2.1. There exists an $\varepsilon_{0}>0$ and constants $B, C>0$ such that, for all $0<\varepsilon<\varepsilon_{0}$ and $0 \leq n \leq N$, the first $N+1$ eigenvalues $\lambda_{0}>\ldots>\lambda_{N}$ of (1.13) satisfy:

(a) For $0<\sigma_{U}<a^{2} / 4$,

$$
\lambda_{0} \in\left[\lambda_{0}^{*, \sigma_{U}}-C \varepsilon^{2 / 3} \mathrm{e}^{-B / \sqrt{\varepsilon}}, \lambda_{0}^{*, \sigma_{L}}+C \varepsilon^{2 / 3} \mathrm{e}^{-B / \sqrt{\varepsilon}}\right]
$$

and

$$
\lambda_{n} \in\left[\lambda_{n}^{*, \sigma_{U}}-C \varepsilon^{1 / 6} \mathrm{e}^{-B / \sqrt{\varepsilon}}, \lambda_{n}^{*, \sigma_{L}}+C \varepsilon^{1 / 6} \mathrm{e}^{-B / \sqrt{\varepsilon}}\right], \quad \text { for all } \quad 1 \leq n \leq N .
$$

(b) For $\sigma_{L}>a^{2} / 4$,

$$
\lambda_{n} \in\left[\lambda_{n+1}^{*, \sigma_{U}}-C \varepsilon^{1 / 6} \mathrm{e}^{-B / \sqrt{\varepsilon}}, \lambda_{n+1}^{*, \sigma_{L}}+C \varepsilon^{1 / 6} \mathrm{e}^{-B / \sqrt{\varepsilon}}\right], \quad \text { for all } \quad 0 \leq n \leq N .
$$

This theorem is proved in Sections 3, 4, and 5 .

Theorem 2.1 establishes that all first $N+1$ eigenvalues of $(1.13)$ are $\mathcal{O}\left(\varepsilon^{1 / 3}\right)$ close to $\lambda^{*}$ in $(2.4)$, except for the special eigenvalue $\lambda_{0}$ if $\sigma_{U}<a^{2} / 4$. Moreover, up to exponentially small terms, the bounds on the eigenvalues are explicitly given in terms of zeroes of the Airy functions $\operatorname{Ai}(x)$ and $\operatorname{Bi}(x)$ (and their derivatives (2.3)) and of the bounds $\sigma_{L} x$ and $\sigma_{U} x$ on $F(x)$ in (2.2), which can be determined also explicitly.

LEMMA 2.1. Let

$$
j_{H}^{(1)}(\kappa)=\frac{\mathrm{e}^{-\kappa}-1+\kappa}{\mathrm{e}^{\kappa}-1-\kappa} \quad \text { and } \quad j_{H}^{(2)}(\kappa)=\frac{\mathrm{e}^{-\kappa}}{j_{H}^{(1)}(\kappa)},
$$

so that $0<j_{H}^{(1)}(\kappa)<j_{H}^{(2)}(\kappa)<1$ for all $\kappa>0$. Also, define, for all $\kappa>0$ and $j_{H} \in\left(j_{H}^{(1)}(\kappa), 1\right)$, the point $x_{0}=x_{0}\left(\kappa, j_{H}\right) \in(0,1)$ via $F\left(x_{0}\right)=x_{0} F^{\prime}\left(x_{0}\right)$. Then,

$$
\sigma_{L}\left(\kappa, j_{H}, \eta_{H}\right)=\left\{\begin{array}{rr}
F^{\prime}(0), & 0<j_{H} \leq j_{H}^{(2)} \\
F(1), & j_{H}>j_{H}^{(2)}
\end{array}, \quad \sigma_{U}\left(\kappa, j_{H}, \eta_{H}\right)= \begin{cases}F(1), & 0<j_{H} \leq j_{H}^{(1)} \\
F^{\prime}\left(x_{0}\right), & j_{H}^{(1)}<j_{H}<1 \\
F^{\prime}(0), & j_{H} \geq 1\end{cases}\right.
$$



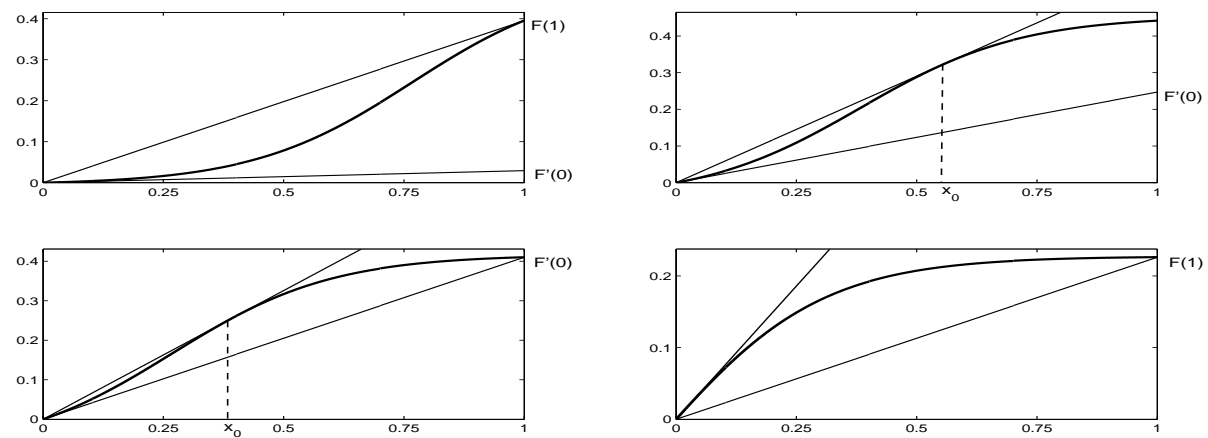

FIG. 2.2. The function $F$ (thick curve) and the linear functions bounding it (thin lines). Here, $\eta_{H}=1, \kappa=6$, and $j_{H}=0.01<j_{H}^{(1)}$ (top left panel), $j_{H}^{(1)}<j_{H}=0.1<j_{H}^{(2)}$ (top right panel), $j_{H}^{(2)}<j_{H}=0.2<1$ (bottom left panel), and $j_{H}=1.2>1$ (bottom right panel).

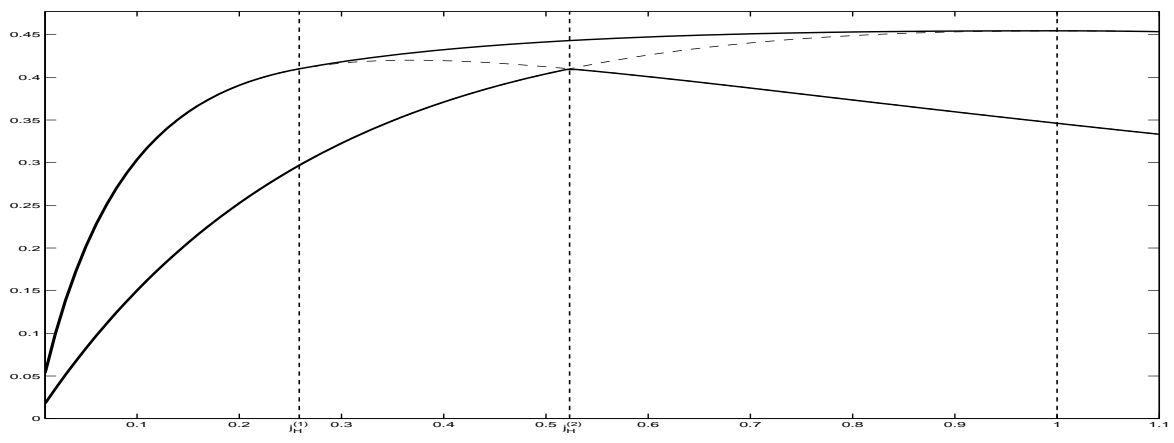

FIG. 2.3. The quantities $\sigma_{U}$ (upper thick curve), $\sigma_{L}$ (lower thick curve), $F(1)$ (dashed curve to the left), and $F^{\prime}(0)$ (dashed curve to the right) as functions of $j_{H}$ and for $\eta_{H}=0.1, \kappa=2$. Note that $F(1)$ merges with $\sigma_{U}$ for $j_{H} \leq j_{H}^{(1)}$ and with $\sigma_{L}$ for $j_{H} \geq j_{H}^{(2)}$, while $F^{\prime}(0)$ merges with $\sigma_{L}$ for $j_{H} \leq j_{H}^{(2)}$ and with $\sigma_{U}$ for $j_{H} \geq 1$. Also note that the WKB method (see Section 6) yields that the location of the eigenvalue close to $\lambda_{0}^{*, \sigma}$ (see Theorem 2.1) is determined, at leading order, by $F(1)$, whereas the location of the eigenvalues close to $\lambda_{n}^{*, \sigma}, n \in \mathbf{N}$, is determined, at leading order, by $F^{\prime}(0)$.

and

$$
\sigma_{L, U}\left(\kappa, j_{H}, \eta_{H}\right)=\nu \sigma_{L, U}\left(\kappa, j_{H}, 0\right), \quad \text { with } \quad \nu=\left(1+\eta_{H}\right)^{-1}
$$

This lemma is proved by straightforward calculus. Figures 2.2 and 2.3 give a graphical representation of the lemma for various representative subcases.

As we shall see in Section 7, the bounds on the eigenvalues established by Theorem 2.1 are quite sharp and agree very well with the bifurcations of the full (unscaled) model (1.1). Nevertheless, the rigorous analysis of Sections 3, 4, and 5 does not give information on the structure of the associated eigenfunctions of (1.13), which is of particular interest to the nature of the patterns that are generated by (1.1) as $\lambda_{0}$ crosses through zero (see Section 7). Moreover, the width of the intervals that bound the eigenvalues $\lambda_{n}$ of (1.13) is of the same order in $\varepsilon$-namely of $\mathcal{O}\left(\varepsilon^{1 / 3}\right)$ - as the distance between successive eigenvalues. This is especially relevant in the transitional case in which $\sigma_{L}<a^{2} / 4<\sigma_{U}$, for which Theorem 2.1 does not give any information. 
Therefore, we complete our analysis of (1.13) by performing an asymptotic WKB approximation in Section 6. We derive asymptotic formulas for the eigenvalues and for the corresponding eigenfunctions. Using these formulas, we show that

- In case (a) of Theorem 2.1, the profile of the eigenfunction $\omega_{0}$, which corresponds to the largest eigenvalue $\lambda_{0}$, is of boundary layer type near the bottom. In terms of the phytoplankton concentration, this profile corresponds to a BL.

- In case (b) of the same theorem, $\omega_{0}$ has the shape of a spike around the point $x=x_{\mathrm{DCM}}$, where $x_{\mathrm{DCM}}$ is determined, to leading order in $\varepsilon$, by $F\left(x_{\mathrm{DCM}}\right)=a^{2} / 4$ (see Fig. 6.1 ). This profile corresponds to a DCM around $x_{\mathrm{DCM}}$.

- The transitional region between cases (a) and (b) in Theorem 2.1 is described, to leading order in $\varepsilon$, by the equation $a^{2} / 4=F(1)$. Indeed, the leading order approximation of $\lambda_{0}$ is $\lambda_{0}=f(1)-\ell$ in the region $F(1)<a^{2} / 4$ (and $\omega_{0}$ is a BL) and $\lambda_{0}=f(0)-\ell-a^{2} / 4$ in the region $F(1)>a^{2} / 4$ (and $\omega_{0}$ is a DCM). Recalling Lemma 2.1, we see that this transition occurs at a value of $a^{2} / 4$ which is (always to leading order in $\varepsilon$ ) equal to $\sigma_{U}$, when $0<j_{H} \leq j_{H}^{(1)}$, equal to $\sigma_{L}$, when $j_{H} \geq j_{H}^{(2)}$, and between $\sigma_{U}$ and $\sigma_{L}$, when $j_{H}^{(1)}<j_{H}<j_{H}^{(2)}$.

3. Eigenvalue bounds . As a first step towards the proof of Theorem 2.1, we recast (1.13) in a form more amenable to analysis. First, we observe that the operator involved in this eigenvalue problem is self-adjoint only if $a=0$. Applying the Liouville transformation

$$
w(x)=\mathrm{e}^{-a x / 2 \sqrt{\varepsilon}} \omega(x)
$$

we obtain the self-adjoint problem

$$
\begin{aligned}
\varepsilon w_{x x}+\left(f(x)-\ell-\frac{a^{2}}{4}\right) w & =\lambda w \\
\left(\sqrt{\varepsilon} w_{x}-\frac{a}{2} w\right)(0)=\left(\sqrt{\varepsilon} w_{x}-\frac{a}{2} w\right)(1) & =0
\end{aligned}
$$

Recalling (2.1) and (2.4), we write this equation in the form

$$
\mathcal{L} w=\mu w, \quad \text { with } \quad \mathcal{G}(w, 0)=\mathcal{G}(w, 1)=0 .
$$

The operator $\mathcal{L}$, the scalar $\mu$, and the linear functionals $\mathcal{G}(\cdot, x)$ are defined by

$$
\mathcal{L}=-\varepsilon \frac{d^{2}}{d x^{2}}+F(x), \quad \mu=\lambda^{*}-\lambda, \quad \mathcal{G}(w, x)=w(x)-\frac{2 \sqrt{\varepsilon}}{a} w_{x}(x) .
$$

This is the desired form of the eigenvalue problem (1.13). To prove Theorem 2.1, we decompose the operator $\mathcal{L}$ into a self-adjoint part for which the eigenvalue problem is solvable and a positive definite part. Then, we use the following comparison principle to obtain the desired bounds.

TheOrem 3.1. ([19, Sections 8.12-8.13]) Let the operators $\hat{\mathcal{A}}$ and $\mathcal{A}$ be self-adjoint, bounded below, and have compact inverses, and write their eigenvalues as $\hat{\mu}_{0} \leq \hat{\mu}_{1} \leq \ldots \leq \hat{\mu}_{n} \leq \ldots$ and $\mu_{0} \leq \mu_{1} \leq \ldots \leq \mu_{n} \leq \ldots$, respectively. If the difference $\mathcal{A}-\hat{\mathcal{A}}$ is positive semidefinite, then $\hat{\mu}_{n} \leq \mu_{n}$, for all $n \in\{0,1, \ldots\}$.

3.1. Crude bounds for the eigenvalues of $\mathcal{L}$. First, we derive crude bounds for the spectrum $\left\{\mu_{n}\right\}$ of $\mathcal{L}$ so as to demonstrate the method and to establish that $\mathcal{L}$ satisfies the boundedness condition of Theorem 3.1 .

LEMMA 3.1. The eigenvalues $\mu_{n}$ satisfy the inequalities

$$
-a^{2} / 4 \leq \mu_{0} \leq F(1)-a^{2} / 4 \quad \text { and } \quad \varepsilon n^{2} \pi^{2} \leq \mu_{n} \leq F(1)+\varepsilon n^{2} \pi^{2}, \quad n \in \mathbf{N} .
$$


Proof. Let $c \in \mathbf{R}$. We start by decomposing $\mathcal{L}$ as

$$
\mathcal{L}=\mathcal{L}^{0, c}+\mathcal{F}^{0, c}, \quad \text { where } \quad \mathcal{L}^{0, c}=-\varepsilon \frac{d^{2}}{d x^{2}}+c \quad \text { and } \quad \mathcal{F}^{0, c}=F(x)-c .
$$

Then, we write $\left\{\mu_{n}^{0, c}\right\}$ for the set of eigenvalues of the problem

$$
\mathcal{L}^{0, c} w^{0, c}=\mu^{0, c} w^{0, c}, \quad \text { with } \quad \mathcal{G}\left(w^{0, c}, 0\right)=\mathcal{G}\left(w^{0, c}, 1\right)=0,
$$

with the eigenvalues arranged so that $\mu_{0}^{0, c} \leq \mu_{1}^{0, c} \leq \ldots \leq \mu_{n}^{0, c} \leq \ldots$.

For $c=c_{L}=0$, the operator $\mathcal{L}^{0, c_{L}}$ is self-adjoint, while $\mathcal{F}^{0, c_{L}}=F(x) \geq 0$ is a positive definite multiplicative operator. Thus, using Theorem 3.1, we obtain the inequalities

$$
\mu_{n}^{0, c_{L}} \leq \mu_{n}, \quad \text { for all } \quad n \in \mathbf{N} \cup\{0\} .
$$

Next, for $c=c_{U}=F(1)$, the operator $\mathcal{F}^{0, c_{U}}=F(x)-F(1) \leq 0$ is negative definite, while $\mathcal{L}^{0, c_{U}}$ is self-adjoint. Hence, we write

$$
\mathcal{L}^{0, c_{U}}=\mathcal{L}-\mathcal{F}^{0, c_{U}}
$$

where $-\mathcal{F}^{0, c_{U}}$ is positive definite. The fact that the spectrum $\left\{\mu_{n}\right\}$ of $\mathcal{L}$ is bounded from below by (3.7) allows us to use Theorem 3.1 to bound each $\mu_{n}$ from above,

$$
\mu_{n} \leq \mu_{n}^{0, c_{U}}, \quad \text { for all } n \in \mathbf{N} \cup\{0\}
$$

Combining this bound and (3.7), we obtain

$$
\mu_{n}^{0, c_{L}} \leq \mu_{n} \leq \mu_{n}^{0, c_{U}}, \quad \text { for all } \quad n \in \mathbf{N} \cup\{0\}
$$

Naturally, the eigenvalue problem (3.6) may be solved exactly to obtain

$$
\mu_{0}^{0, c}=c-a^{2} / 4 \quad \text { and } \quad \mu_{n}^{0, c}=c+\varepsilon n^{2} \pi^{2}, \quad n \in \mathbf{N} .
$$

Combining these formulas with (3.8), we obtain the inequalities (3.4).

3.2. Tight bounds for the eigenvalues of $\mathcal{L}$. The accurate bounds for the eigenvalues of (3.2) described in Theorem 2.1 may be obtained by bounding $F$ by linear functions. In the next lemma, we bound the eigenvalues $\mu_{n}$ by the eigenvalues $\mu_{n}^{1, \sigma}$ of a simpler problem. Then, in Lemma 3.3, we obtain strict, exponentially small bounds for $\mu_{n}^{1, \sigma}$. Theorem 2.1 follows readily by combining these two lemmas and using definitions (2.4) and (3.13).

LEMmA 3.2. Let $\sigma \in\left\{\sigma_{L}, \sigma_{U}\right\}$, with $\sigma_{L}$ and $\sigma_{U}$ as defined in (2.5), define the operator $\mathcal{L}^{1, \sigma}=$ $-\varepsilon \frac{d^{2}}{d x^{2}}+\sigma x$, and write $\left\{\mu_{n}^{1, \sigma}\right\}$ for the eigenvalues corresponding to the problem

$$
\mathcal{L}^{1, \sigma} w=\mu^{1, \sigma} w, \quad \text { with } \quad \mathcal{G}(w, 0)=\mathcal{G}(w, 1)=0 .
$$

Let $\left\{\mu_{n}^{1, \sigma}\right\}$ be arranged so that $\mu_{0}^{1, \sigma} \leq \mu_{1}^{1, \sigma} \leq \ldots \leq \mu_{n}^{1, \sigma} \leq \ldots$. Then,

$$
\mu_{n}^{1, \sigma_{L}} \leq \mu_{n} \leq \mu_{n}^{1, \sigma_{U}}, \quad \text { for all } \quad n \in \mathbf{N} \cup\{0\} .
$$


Proof. First, we decompose $\mathcal{L}$ as

$$
\mathcal{L}=\mathcal{L}^{1, \sigma}+\mathcal{F}^{1, \sigma}, \quad \text { where } \quad \mathcal{L}^{1, \sigma}=-\varepsilon \frac{d^{2}}{d x^{2}}+\sigma x, \mathcal{F}^{1, \sigma}=F(x)-\sigma x,
$$

and $\sigma \in\left\{\sigma_{L}, \sigma_{U}\right\}$. We note here that $\mathcal{L}^{1, \sigma}$ is self-adjoint.

Next, $\mathcal{F}^{1, \sigma_{L}}$ is a positive definite multiplicative operator, since $F(x) \geq \sigma_{L} x$ (see (2.2)). Thus, $\mu_{n}^{1, \sigma_{L}} \leq \mu_{n}$, for all $n \in \mathbf{N} \cup\{0\}$, by Theorem 3.1. On the contrary, $\mathcal{F}^{1, \sigma_{U}}$ is negative definite, since $F(x) \leq \sigma_{U} x$. Therefore, we write

$$
\mathcal{L}^{1, \sigma_{U}}=\mathcal{L}-\mathcal{F}^{1, \sigma_{U}}
$$

where now $-\mathcal{F}^{1, \sigma_{U}}$ is positive definite. The fact that the spectrum $\left\{\mu_{n}\right\}$ is bounded from below by Lemma 3.1 allows us to use Theorem 3.1 to bound each $\mu_{n}$ from above, $\mu_{n} \leq \mu_{n}^{1, \sigma_{U}}$. Combining both bounds for each $n$, we obtain (3.11).

Hence, it remains to solve the eigenvalue problem (3.10). Although this problem is not exactly solvable, the eigenvalues may be calculated up to terms exponentially small in $\varepsilon$. In particular, recalling the definitions in Section 2 and letting

$$
\mu_{0}^{*, \sigma}=\lambda^{*}-\lambda_{0}^{*, \sigma}=-\sigma B_{0, \sigma} \quad \text { and } \quad \mu_{n}^{*, \sigma}=\lambda^{*}-\lambda_{n}^{*, \sigma}=\varepsilon^{1 / 3} \sigma^{2 / 3}\left|A_{n, \sigma}^{\prime}\right|>0,
$$

for $n \geq 1$, we can prove the following lemma.

LEMMA 3.3. Let $N \in \mathbf{N}$ be fixed and define

$$
\begin{aligned}
& \delta_{0, \sigma}=\varepsilon^{2 / 3} \sigma^{-2 / 3} \exp \left(-\frac{2}{3}\left[3\left(1+B_{0, \sigma}-B\right)^{3 / 2}-2\left(B_{0, \sigma}-B\right)^{3 / 2}-\left(1+B_{0, \sigma}+B\right)^{3 / 2}\right] \sqrt{\frac{\sigma}{\varepsilon}}\right), \\
& \delta_{n, \sigma}=\varepsilon^{1 / 6} \exp \left(-\frac{4}{3} \sqrt{\frac{\sigma}{\varepsilon}}+2\left|A_{n+1}\right|\left(\frac{\sigma}{\varepsilon}\right)^{1 / 6}\right), \quad \text { for all } 1 \leq n \leq N+1,
\end{aligned}
$$

and for all $0<B<B_{0, \sigma}$ for which the exponent in the expression for $\delta_{0, \sigma}$ is negative. Then, for each such $B$ there is an $\varepsilon_{0}>0$ and positive constants $C_{0}, \ldots, C_{N+1}$, such that, for all $0<\varepsilon<\varepsilon_{0}$ and $0 \leq n \leq N$, the first $N+1$ eigenvalues $\mu_{0}^{1, \sigma}, \ldots, \mu_{N}^{1, \sigma}$ corresponding to (3.10) satisfy:

(a) For $0<\sigma<a^{2} / 4,\left|\mu_{0}^{1, \sigma}-\mu_{0}^{*, \sigma}\right|<C_{0} \delta_{0, \sigma}$ and $\left|\mu_{n}^{1, \sigma}-\mu_{n}^{*, \sigma}\right|<C_{n} \delta_{n, \sigma}$ for all $1 \leq n \leq N$.

(b) For $\sigma>a^{2} / 4,\left|\mu_{n}^{1, \sigma}-\mu_{n+1}^{*, \sigma}\right|<C_{n+1} \delta_{n+1, \sigma}$, for all $0 \leq n \leq N$.

The bounds on the eigenvalues $\mu_{0}^{1, \sigma}, \ldots, \mu_{N}^{1, \sigma}$ are derived in Section 4. The fact that these are indeed the $N+1$ first eigenvalues corresponding to (3.10) is proved in Section 5. Note that Theorem 2.1 follows immediately from this lemma, in combination with the above analysis.

4. The eigenvalues $\mu_{0}^{1, \sigma}, \ldots, \mu_{N}^{1, \sigma}$. In this section, we derive the bounds on $\mu_{0}^{1, \sigma}, \ldots, \mu_{N}^{1, \sigma}$ of Lemma 3.3. In Section 4.1, we reduce the eigenvalue problem (3.10) to the algebraic one of locating the roots of an Evans-type function $\mathcal{D}$. In Section 4.2 , we identify the roots of $\mathcal{D}$ with those of two functions $\mathcal{A}$ and $\mathcal{B}$ which are simpler to analyze. Finally, in Section 4.3, we identify the relevant roots of $\mathcal{A}$ and $\mathcal{B}$ and thus also of $\mathcal{D}$.

4.1. Reformulation of the eigenvalue problem . First, we derive an algebraic equation whose solutions correspond to the eigenvalues of (3.10). We start by rescaling the parameter $a$, the small parameter $\varepsilon$, the eigenvalue $\mu^{1, \sigma}$, and the independent variable $x$ via

$$
\beta=\frac{a}{2 \sqrt{\sigma}}, \quad 0<\gamma \equiv\left(\frac{\varepsilon}{\sigma}\right)^{1 / 3} \ll 1, \quad \bar{\chi}=-\frac{\mu^{1, \sigma}}{\gamma \sigma}, \quad x=\gamma(\chi-\bar{\chi}),
$$



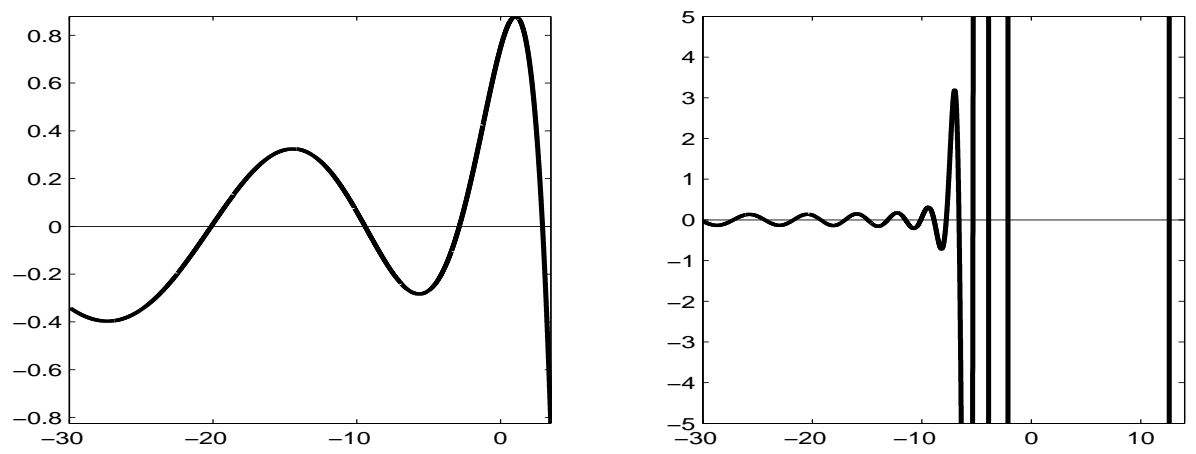

FiG. 4.1. The function $\mathcal{D}(\bar{\chi})$ for $a=3, \sigma=1$, and $\varepsilon=0.1$ (left panel), $\varepsilon=0.001$ (right panel).

and we note that the inequalities $0<\sigma<a^{2} / 4$ and $\sigma>a^{2} / 4$ become $\beta>1$ and $0<\beta<1$, respectively. Then, we define the linear functional

$$
\Gamma(w, \bar{\chi})=w(\bar{\chi})-\frac{\sqrt{\gamma}}{\beta} w^{\prime}(\bar{\chi}), \quad \text { for all differentiable functions } w,
$$

and we remark that, for $w$ equal to $\mathrm{Ai}$ or $\mathrm{Bi}$, this definition agrees with the one given in (2.3). Further introducing the Wronskian

$$
\mathcal{D}(\bar{\chi})=\Gamma(\mathrm{Ai}, \bar{\chi}) \Gamma\left(\mathrm{Bi}, \gamma^{-1}+\bar{\chi}\right)-\Gamma\left(\mathrm{Ai}, \gamma^{-1}+\bar{\chi}\right) \Gamma(\mathrm{Bi}, \bar{\chi})
$$

(see also Fig. 4.1), we can prove the following lemma.

Lemma 4.1. The eigenvalue problem (3.10) has $\mu^{1, \sigma}$ as an eigenvalue if and only if $\mathcal{D}(\bar{\chi})=0$.

Proof. Using (4.1), we rewrite problem (3.10) in the form

$$
\begin{aligned}
\frac{d^{2} w}{d \chi^{2}} & =\chi w, \quad \chi \in\left[\bar{\chi}, \gamma^{-1}+\bar{\chi}\right], \\
\Gamma(w, \bar{\chi})=\Gamma\left(w, \gamma^{-1}+\bar{\chi}\right) & =0 .
\end{aligned}
$$

This is an Airy equation and thus has the general solution

$$
w(\chi)=D_{A} \operatorname{Ai}(\chi)+D_{B} \operatorname{Bi}(\chi) .
$$

The boundary conditions become

$$
\begin{aligned}
& \Gamma(w, \bar{\chi})=D_{A} \Gamma(\mathrm{Ai}, \bar{\chi})+D_{B} \Gamma(\mathrm{Bi}, \bar{\chi})=0, \\
& \Gamma\left(w, \gamma^{-1}+\bar{\chi}\right)=D_{A} \Gamma\left(\mathrm{Ai}, \gamma^{-1}+\bar{\chi}\right)+D_{B} \Gamma\left(\mathrm{Bi}, \gamma^{-1}+\bar{\chi}\right)=0 .
\end{aligned}
$$

The sufficient and necessary condition for the existence of nontrivial solutions to this system is that its determinant, which is the Wronskian $\mathcal{D}$ given in (4.3), vanishes, and the lemma is proved.

4.2. Product decomposition of the function $\mathcal{D}$. In the preceding section, we saw that the values of $\bar{\chi}$ that correspond to the eigenvalues $\mu^{1, \sigma}$ must be zeroes of $\mathcal{D}$. In the next section, we will prove that the first few zeroes of $\mathcal{D}$ are all $\mathcal{O}(1)$, in the case $0<\beta<1$, and both $\mathcal{O}(1)$ and $\mathcal{O}\left(\gamma^{-1}\right)$ in the case $\beta>1$. To identify them, we rewrite $\mathcal{D}$ in the form

$$
\mathcal{D}(\bar{\chi})=\Gamma\left(\mathrm{Bi}, \gamma^{-1}+\bar{\chi}\right) \mathcal{A}(\bar{\chi})=\Gamma(\mathrm{Ai}, \bar{\chi}) \mathcal{B}(\bar{\chi}),
$$


where we have defined the functions

$$
\begin{aligned}
& \mathcal{A}(\bar{\chi})=\Gamma(\mathrm{Ai}, \bar{\chi})-\frac{\Gamma\left(\mathrm{Ai}, \gamma^{-1}+\bar{\chi}\right)}{\Gamma\left(\mathrm{Bi}, \gamma^{-1}+\bar{\chi}\right)} \Gamma(\mathrm{Bi}, \bar{\chi}), \\
& \mathcal{B}(\bar{\chi})=\Gamma\left(\mathrm{Bi}, \gamma^{-1}+\bar{\chi}\right)-\frac{\Gamma(\mathrm{Bi}, \bar{\chi})}{\Gamma(\mathrm{Ai}, \bar{\chi})} \Gamma\left(\mathrm{Ai}, \gamma^{-1}+\bar{\chi}\right) .
\end{aligned}
$$

Here, $\mathcal{A}$ is well-defined for all $\bar{\chi}$ such that $\Gamma\left(\mathrm{Bi}, \gamma^{-1}+\bar{\chi}\right) \neq 0$, while $\mathcal{B}$ is well-defined for all $\bar{\chi}$ such that $\Gamma(\mathrm{Ai}, \bar{\chi}) \neq 0$. Equation (4.7) implies that the roots of $\mathcal{A}$ and $\mathcal{B}$ are also roots of $\mathcal{D}$.

In the next section, we will establish that the $\mathcal{O}(1)$ roots of $\mathcal{D}$ coincide with roots of $\mathcal{A}$ and the $\mathcal{O}\left(\gamma^{-1}\right)$ ones with roots of $\mathcal{B}$. To prove this, we first characterize the behaviors of $\mathcal{A}$ and $\mathcal{B}$ for $\mathcal{O}(1)$ and $\mathcal{O}\left(\gamma^{-1}\right)$ values of $\bar{\chi}$, respectively, by means of the next two lemmas. In what follows, we write $E(x)=\exp \left(-(2 / 3) x^{3 / 2}\right)$ for brevity and $\|\cdot\|_{\left[X_{L}, X_{R}\right]}$ for the $\mathrm{W}_{\infty}^{1}-$ norm over any interval $\left[X_{L}, X_{R}\right]$,

$$
\|w\|_{\left[X_{L}, X_{R}\right]}=\max _{\bar{\chi} \in\left[X_{L}, X_{R}\right]}|w(\bar{\chi})|+\max _{\bar{\chi} \in\left[X_{L}, X_{R}\right]}\left|w^{\prime}(\bar{\chi})\right| .
$$

Lemma 4.2. Let $X<0$ be fixed. Then, there is a $\gamma_{0}>0$ and a constant $c_{A}>0$ such that

$$
\|\mathcal{A}(\bar{\chi})-\Gamma(\mathrm{Ai}, \bar{\chi})\|_{[X, 0]}<c_{A} \gamma^{-1 / 2} E\left(\gamma^{-1}(2+3 X \gamma)^{2 / 3}\right), \quad \text { for all } 0<\gamma<\gamma_{0} .
$$

For the next lemma, we switch to the independent variable $\bar{\psi}=\gamma \bar{\chi}$ to facilitate calculations. We analyze the behavior of $\mathcal{B}\left(\gamma^{-1} \bar{\psi}\right)$ for $\mathcal{O}(1)$ values of $\bar{\psi}$ (equivalently, for $\mathcal{O}\left(\gamma^{-1}\right)$ values of $\bar{\chi}$ ) as $\gamma \downarrow 0$.

Lemma 4.3. Let $0<\Psi_{L}<\Psi_{R}$ be fixed. Then, there is a $\gamma_{0}>0$ and a constant $c_{B}>0$ such that, for all $0<\gamma<\gamma_{0}$,

$$
\|\left. E\left(\gamma^{-1}(1+\bar{\psi})\right)\left[\mathcal{B}\left(\gamma^{-1} \bar{\psi}\right)-\Gamma\left(\mathrm{Bi}, \gamma^{-1}(1+\bar{\psi})\right)\right]\right|_{\left[\Psi_{L}, \Psi_{R}\right]}<c_{B} \gamma^{-1 / 4}\left[\frac{E\left(\gamma^{-1}\left(1+\Psi_{L}\right)\right)}{E\left(\gamma^{-1} \Psi_{L}\right)}\right]^{2} .
$$

The proofs of these lemmas are given in Appendices B and C, respectively.

4.3. Zeroes of $\mathcal{D}$. Using Lemma 4.2 and an auxiliary result, we can locate the roots of $\mathcal{D}$.

Lemma 4.4. Let $N \in \mathbf{N}$ be fixed, $A_{n, \sigma}^{\prime}$ and $B_{0, \sigma}$ be defined as in Section 2, and $B, \delta_{0, \sigma}, \ldots, \delta_{N, \sigma}$ as in Lemma 3.3. Then, for each admissible $B$, there is a $\gamma_{0}>0$ and positive constants $c_{0}, \ldots, c_{n}$ such that, for all $0<\gamma<\gamma_{0}$, the function $\mathcal{D}(\bar{\chi})$ has roots $\bar{\chi}_{0}>\bar{\chi}_{1}>\ldots>\bar{\chi}_{N}$ which satisfy the following bounds:

(a) For $\beta>1$ (equivalently, for $0<\sigma<a^{2} / 4$ ),

$$
\left|\bar{\chi}_{0}-\gamma^{-1} B_{0, \sigma}\right|<c_{0} \gamma^{-1} \delta_{0, \sigma} \quad \text { and } \quad\left|\bar{\chi}_{n}-A_{n, \sigma}^{\prime}\right|<c_{n} \gamma^{-1} \delta_{n, \sigma}, \quad \text { for all } \quad 1 \leq n \leq N .
$$

(b) For $0<\beta<1$ (equivalently, for $\sigma>a^{2} / 4$ ),

$$
\left|\bar{\chi}_{n}-A_{n+1, \sigma}^{\prime}\right|<c_{n} \gamma^{-1} \delta_{n+1, \sigma}, \quad \text { for all } \quad 0 \leq n \leq N .
$$

The proof of this lemma requires the following elementary result.

Lemma 4.5. Let $C, G$, and $H$ be real-valued, continuous functions. Let $\delta>0$ and $z_{0} \in$ $\left[Z_{L}, Z_{R}\right] \subset \mathbf{R}$ be such that

$$
H\left(z_{0}\right)=0, \quad \max _{\left[Z_{L}, Z_{R}\right]} H^{\prime}=-H_{0}<0, \quad \max _{\left[Z_{L}, Z_{R}\right]}|C(G-H)|<\delta \quad \text { and } \quad \min _{\left[Z_{L}, Z_{R}\right]} C=C_{0}>0 .
$$


If $\delta<C_{0} H_{0} \min \left(z_{0}-Z_{L}, Z_{R}-z_{0}\right)$, then $G$ has a zero $z_{*}$ such that $\left|z_{*}-z_{0}\right| \leq \delta /\left(C_{0} H_{0}\right)$.

Proof. Let $z_{\ell}=z_{0}-\delta /\left(C_{0} H_{0}\right)$ and $z_{r}=z_{0}+\delta /\left(C_{0} H_{0}\right)$. By assumption, $Z_{L}<z_{\ell}<z_{0}<z_{r}<Z_{R}$, and thus

$G\left(z_{\ell}\right)=H\left(z_{\ell}\right)+G\left(z_{\ell}\right)-H\left(z_{\ell}\right) \geq \int_{z_{0}}^{z_{\ell}} H^{\prime}(z) d z-\frac{\max _{\left[Z_{L}, Z_{R}\right]}|C(G-H)|}{\min _{\left[Z_{L}, Z_{R}\right]} C}>\left(z_{0}-z_{\ell}\right) H_{0}-\frac{\delta}{C_{0}}=0$.

Similarly, we may prove that $G\left(z_{r}\right)<0$ and the desired result follows.

Proof of Lemma 4.4 (a) First, we prove the existence of a root $\bar{\chi}_{0}$ satisfying the desired bound. We start by rescaling the independent variable through $\bar{\psi}=\gamma \bar{\chi}$. Then, it suffices to show that there is a root $\bar{\psi}_{0}$ of $\mathcal{D}\left(\gamma^{-1} \bar{\psi}\right)$ satisfying the bound $\left|\bar{\psi}_{0}-B_{0, \sigma}\right|<c_{0} \delta_{0}$, for some $c_{0}>0$. Equation (4.7) reads $\mathcal{D}\left(\gamma^{-1} \bar{\psi}\right)=\Gamma\left(\mathrm{Ai}, \gamma^{-1} \bar{\psi}\right) \mathcal{B}\left(\gamma^{-1} \bar{\psi}\right)$. Here, $\Gamma\left(\mathrm{Ai}, \gamma^{-1} \bar{\psi}\right)$ has no positive roots, by definition of $\Gamma$ and because $\operatorname{Ai}\left(\gamma^{-1} \bar{\psi}\right)>0$ and $\operatorname{Ai}^{\prime}\left(\gamma^{-1} \bar{\psi}\right)<0$, for all $\bar{\psi}>0$. Thus, $\bar{\chi}_{0}$ must be a root of $\mathcal{B}$. Its existence and the bound on it follow from Lemmas 4.3 and 4.5. Indeed, let $z_{0}=B_{0, \sigma}, Z_{L}=B_{0, \sigma}-B$, $Z_{R}=B_{0, \sigma}+B, C=E$ (see Section 4.2$), G=B$, and $H=\Gamma(\mathrm{Bi}, \cdot)$. Lemma 4.3 provides a bound $\delta$ on $\|C(G-H)\|_{\left[Z_{L}, Z_{R}\right]}$. Also, using Corollary A.1, we may calculate

$$
\begin{aligned}
C_{0} & =\min _{\left[Z_{L}, Z_{R}\right]} E\left(\gamma^{-1}(1+\bar{\psi})\right)=E\left(\gamma^{-1}\left(1+Z_{R}\right)\right), \\
-H_{0} & =\max _{\left[Z_{L}, Z_{R}\right]} \Gamma\left(\mathrm{Bi}^{\prime}, \gamma^{-1}(1+\bar{\psi})\right)<-c \gamma^{5 / 4}\left[E\left(\gamma^{-1}\left(1+Z_{L}\right)\right)\right]^{-1} .
\end{aligned}
$$

Now, $\delta$ satisfies the condition $\delta<C_{0} H_{0} B$ of Lemma 4.5 for all $\gamma$ small enough. Thus, we may apply Lemma 4.5 to obtain the desired bound on $\bar{\chi}_{0}$.

Next, we show that $\mathcal{A}$ has the remaining roots $\bar{\chi}_{1}, \ldots, \bar{\chi}_{N}$. We fix $A_{N+1}<X<A_{N}$ and let $I_{1}, \ldots, I_{N}$ be disjoint intervals around $A_{1}, \ldots, A_{N}$, respectively. Lemma 4.2 states that $\mathcal{A}(\bar{\chi})$ and $\Gamma(\mathrm{Ai}, \bar{\chi})$ are exponentially close in the $\mathrm{W}_{\infty}^{1}-$ norm over $[X, 0]$. Thus, for all $0<\gamma<\gamma_{0}$ (with $\gamma_{0}$ small enough), $\mathcal{A}$ has $N$ distinct roots $\bar{\chi}_{1} \in I_{1}, \ldots, \bar{\chi}_{N} \in I_{N}$ in $[X, 0]$ by Lemma A.2. Since $\Gamma\left(\mathrm{Bi}, \gamma^{-1}+\bar{\chi}\right)$ can be bounded away from zero over $[X, 0]$ using Lemma A.1 (with $p=1$ and $q=\bar{\chi}$ ), we conclude that $\mathcal{D}$ has the $N$ distinct roots $\bar{\chi}_{1}, \ldots, \bar{\chi}_{N}$ in $[X, 0]$.

(b) The argument used in part (a) - where $\beta>1$ - to establish the bounds on the $\mathcal{O}(1)$ roots of $\mathcal{A}$ does not depend on the sign of $\beta-1$. Therefore, it applies also to this case - where $0<\beta<1-$, albeit in an interval $[X, 0]$, with $A_{N+2}<X<A_{N+1}$, yielding $N+1$ roots which we label $\bar{\chi}_{0}, \ldots, \bar{\chi}_{N}$.

On the other hand, $B_{0, \sigma}<0$ for $0<\beta<1$, because of the estimate on $B_{0, \sigma}$ in Lemma A.2. As a result, the argument used to identify that root does not apply any more, since now $B_{0, \sigma}<0$ and thus Lemma 4.3 may not be applied to provide the bound $\delta$ needed in Lemma 4.5 . In fact, were this root to persist and remain close to $\gamma^{-1} B_{0, \sigma}$ as in case (a), it would become large and negative by the estimate in Lemma A.2 and hence smaller than the roots $\bar{\chi}_{0}, \ldots, \bar{\chi}_{N}$ obtained above. Thus, it could never be the leading eigenvalue in this parameter regime.

5. The eigenfunctions $w_{0}^{1, \sigma}, \ldots, w_{N}^{1, \sigma}$. In the previous section, we located some of the eigenvalues $\mu^{1, \sigma}$. In this section, we show that the eigenvalues we identified are the largest ones. To achieve this, we derive formulas for the eigenfunctions $w_{0}^{1, \sigma}, \ldots, w_{N}^{1, \sigma}$ associated with $\mu_{0}^{1, \sigma}, \ldots, \mu_{N}^{1, \sigma}$, respectively, and show that $w_{n}^{1, \sigma}$ has $n$ zeroes in the interval $\left[\bar{\chi}_{n}, \gamma^{-1}+\bar{\chi}_{n}\right]$ (corresponding to the interval $[0,1]$ in terms of $\chi$, see (4.1)). The desired result follows, then, from standard Sturm-Liouville theory [4]. In particular, we prove the following lemma.

Lemma 5.1. Let $N \in \mathbf{N}$. Then, there is a $\gamma_{0}>0$ such that, for all $0<\gamma<\gamma_{0}$ and for all $n=0,1, \ldots, N$, the eigenfunction $w_{n}^{1, \sigma}$ corresponding to the eigenvalue $\mu_{n}^{1, \sigma}$ has exactly $n$ zeroes in the interval $\left[\bar{\chi}_{n}, \gamma^{-1}+\bar{\chi}_{n}\right]$. 
The proof of this lemma occupies the rest of this section. Parallel to it, we show that the profile of $\omega_{0}$ associated with $w_{0}$ through (3.1) (a) is that of a boundary layer near the bottom of the water column, for $\beta>1$ (BL) and (b) is that of an interior, non-monotone boundary layer (a spike [9]) close to the point $0<x_{\mathrm{DCM}}=\beta^{2}<1$, for $0<\beta<1$ (DCM).

We start by fixing $\bar{\chi}$ to be $\bar{\chi}_{n}$, for some $n=1, \ldots, N$. The corresponding eigenvalue is $\mu_{n}^{1, \sigma}=$ $-\gamma \sigma \bar{\chi}_{n}$ (see (4.1)), while the corresponding eigenfunction $w_{n}$ is given by (4.5),

$$
w_{n}^{1, \sigma}(\chi)=D_{A} \operatorname{Ai}(\chi)+D_{B} \operatorname{Bi}(\chi), \quad \text { where } \chi \in\left[\bar{\chi}_{n}, \gamma^{-1}+\bar{\chi}_{n}\right] .
$$

Here, the coefficients $D_{A}$ and $D_{B}$ satisfy (4.6),

$$
D_{A} \Gamma_{L, n}(\mathrm{Ai})+D_{B} \Gamma_{L, n}(\mathrm{Bi})=D_{A} \Gamma_{R, n}(\mathrm{Ai})+D_{B} \Gamma_{R, n}(\mathrm{Bi})=0
$$

where $\Gamma_{L, n}(\cdot)=\Gamma\left(\cdot, \bar{\chi}_{n}\right), \Gamma_{R, n}(\cdot)=\Gamma\left(\cdot, \gamma^{-1}+\bar{\chi}_{n}\right)$. We treat the cases $\beta>1$ and $\beta<1$ separately.

5.1. The case $\beta>1$. In this section, we select $D_{A}$ and $D_{B}$ so that (5.1) becomes

$$
w_{n}^{1, \sigma}(\chi)=D_{n} \operatorname{Bi}(\chi)-\operatorname{Ai}(\chi), \quad \text { with } D_{n}=\frac{\Gamma_{L, n}(\mathrm{Ai})}{\Gamma_{L, n}(\mathrm{Bi})}=\frac{\Gamma_{R, n}(\mathrm{Ai})}{\Gamma_{R, n}(\mathrm{Bi})}
$$

Using this formula, we prove Lemma 5.1 and verify that $\omega_{0}$ is of boundary layer type near $x=1$.

5.1.1. The eigenfunction $w_{0}^{1, \sigma}$. First, we show that $w_{0}^{1, \sigma}$ has no zeroes in the corresponding interval. Using Lemma A.1 and the estimates of Lemmas 4.4 for $\bar{\chi}_{0}$ and A.2 for $B_{0, \sigma}$, we estimate

$$
D_{0}=\left(\frac{\Delta_{1}^{2}}{2}+\bar{C}_{0}(\gamma)\right) \exp \left(-4\left(\frac{\left(\beta^{2}-1\right)^{3 / 4}}{3 \gamma^{3 / 2}}+\sqrt{1-\frac{1}{\beta^{2}}}\right)\right) .
$$

Here, $\Delta_{1}^{2}=\left(\beta+\sqrt{\beta^{2}-1}\right) /\left(\beta-\sqrt{\beta^{2}-1}\right)$ and $\left|\bar{C}_{0}(\gamma)\right|<c_{0} \sqrt{\gamma}$, for some $c_{0}>0$. Thus also, $D_{0}>0$.

It suffices to show that $w_{0}^{1, \sigma}$ is positive in this interval, and thus that $\left(w_{0}^{1, \sigma}\right)^{\prime}>0$ everywhere on the interval and $w_{0}^{1, \sigma}\left(\bar{\chi}_{0}\right)>0$. For $n=0,(5.2)$ yields $\left(w_{0}^{1, \sigma}\right)^{\prime}(\chi)=D_{0} \operatorname{Bi}^{\prime}(\chi)-\operatorname{Ai}^{\prime}(\chi)$, while Lemma 4.4 shows that $\left[\bar{\chi}_{0}, \gamma^{-1}+\bar{\chi}_{0}\right] \subset \mathbf{R}_{+}$. Hence, $\operatorname{Bi}^{\prime}(\chi)>0$ and $\operatorname{Ai}^{\prime}(\chi)<0$ for all $\chi$ in this interval. Since $D_{0}>0$, we conclude that $\left(w_{0}^{1, \sigma}\right)^{\prime}>0$, as desired. Next, we determine the sign of $w_{0}^{1, \sigma}\left(\bar{\chi}_{0}\right)$ The function $w_{0}^{1, \sigma}\left(\bar{\chi}_{0}\right)$ is given in (5.2) with $n=0$, while the definition of $\Gamma_{L, 0}$ yields

$$
\operatorname{Ai}\left(\bar{\chi}_{0}\right)=\Gamma_{L, 0}(\mathrm{Ai})+\beta^{-1} \sqrt{\gamma} \operatorname{Ai}^{\prime}\left(\bar{\chi}_{0}\right) \text { and } \operatorname{Bi}\left(\bar{\chi}_{0}\right)=\Gamma_{L, 0}(\operatorname{Bi})+\beta^{-1} \sqrt{\gamma} \operatorname{Bi}^{\prime}\left(\bar{\chi}_{0}\right) .
$$

Substituting in (5.2), we calculate $w_{0}^{1, \sigma}\left(\bar{\chi}_{0}\right)=\beta^{-1} \gamma^{1 / 2}\left[D_{0} \operatorname{Bi}^{\prime}\left(\bar{\chi}_{0}\right)-\operatorname{Ai}^{\prime}\left(\bar{\chi}_{0}\right)\right]$. Thus, $w_{0}^{1, \sigma}\left(\bar{\chi}_{0}\right)$ is positive by our remarks on the signs of $\mathrm{Bi}^{\prime}, \mathrm{Ai}^{\prime}$, and $D_{0}$, and the proof is complete.

Next, we study the profile of the associated solution $\omega_{0}$ to the original problem (1.13). Equations (3.1) and (4.1) yield

$$
\omega_{0}(x)=\exp \left(\frac{\beta}{\gamma^{3 / 2}} x\right)\left[D_{0} \operatorname{Bi}\left(\gamma^{-1} x+\bar{\chi}_{0}\right)-\operatorname{Ai}\left(\gamma^{-1} x+\bar{\chi}_{0}\right)\right], \quad x \in[0,1] .
$$

Using the estimation of Lemma 4.4 for $\bar{\chi}_{0}$ and the estimations of Lemma A.1 for Ai and Bi, we find

$$
\omega_{0}(x)=C_{I}(x)\left(x+\beta^{2}-1\right)^{-1 / 4} \exp \left(\frac{\beta}{\gamma^{3 / 2}} x\right) \sinh \left(\theta_{1}(x)\right), \quad x \in[0,1],
$$

where $C_{I}(x)=C_{I, 0}+C_{I, 1}(x), \sup _{[0,1]}\left|C_{I, 1}(x)\right|<c_{I} \sqrt{\gamma}$, for some $c_{I}>0$, and

$$
\theta_{1}(x)=\frac{2}{3 \gamma^{3 / 2}}\left[\left(x+\beta^{2}-1\right)^{3 / 2}-\left(\beta^{2}-1\right)^{3 / 2}\right]+\frac{2}{\beta}\left[\left(x+\beta^{2}-1\right)^{1 / 2}-\left(\beta^{2}-1\right)^{1 / 2}\right]+\log \Delta_{1} .
$$


The first two terms in the right member of the expression for $\omega_{0}$ are bounded, while the other two correspond to localized concentrations (boundary layers) at $x=1$. Thus, $\omega_{0}$ also corresponds to a boundary layer of width $\mathcal{O}\left(\gamma^{3 / 2}\right)$ at the same point.

5.1.2. The eigenfunctions $w_{1}^{1, \sigma}, \ldots, w_{N}^{1, \sigma}$. Next, we show that the eigenfunction $w_{n}^{1, \sigma}$ has $n$ zeroes in $\left[\bar{\chi}_{n}, \gamma^{-1}+\bar{\chi}_{n}\right]$, where $n=1, \ldots, N$. The eigenfunction $w_{n}^{1, \sigma}$ is given by (5.2). Here also, Lemmas A.1 and 4.4 yield

$$
D_{n}=\left(\frac{\Delta_{2}^{2}}{2}+\bar{C}_{n}(\gamma)\right) \exp \left(-\frac{4}{3 \gamma^{3 / 2}}+2 \frac{\left|A_{n}\right|}{\sqrt{\gamma}}-\frac{2}{\beta}\right),
$$

where $\Delta_{2}^{2}=(\beta+1) /(\beta-1)$ and $\left|\bar{C}_{n}(\gamma)\right|<c_{n} \sqrt{\gamma}$, for some $c_{n}>0$. Hence, $D_{n}>0$.

First, we show that the function $w_{n}^{1, \sigma}$ has exactly $n-1$ zeroes in $\left[\bar{\chi}_{n}, 0\right]$. The estimate (5.3) and the fact that $\mathrm{Bi}$ is uniformly bounded on $\left[\bar{\chi}_{n}, 0\right]$ imply that, for all $0<\gamma<\gamma_{0}$ (with $\gamma_{0}$ small enough), the functions $w_{n}^{1, \sigma}$ and - Ai are exponentially close in the $\mathrm{W}_{\infty}^{1}-$ norm over that interval,

$$
\| w_{n}^{1, \sigma}+\mathrm{Ai}||_{\left[\bar{\chi}_{n}, 0\right]}<c_{n} \exp \left(-\frac{4}{3 \gamma^{3 / 2}}+2 \frac{\left|A_{n}\right|}{\sqrt{\gamma}}\right), \text { for some } c_{n}>0 \text {. }
$$

As a result, we may use an argument exactly analogous to that used in the proof of Lemma 4.4 to show that $w_{n}^{1, \sigma}$ has at least $n-1$ distinct zeroes in that interval, each of which is exponentially close to one of $A_{1}, \ldots, A_{n-1}$. Also, observing that $\bar{\chi}_{n}$ is algebraically larger than $A_{n}$, by Lemmas 4.4 and A.2, while $w_{n}^{1, \sigma}$ is exponentially close to $-\mathrm{Ai}$, by estimate (5.4), we conclude that the zero of $w_{n}^{1, \sigma}$ close to $A_{n}$ lies to the left of $\bar{\chi}_{n}$ and thus there are no other zeroes in $\left[\bar{\chi}_{n}, \gamma^{-1}+\bar{\chi}_{n}\right]$.

It only remains to show that there is a unique zero of $w_{n}^{1, \sigma}$ in $\left[0, \gamma^{-1}+\bar{\chi}_{n}\right]$. We work as in Section 5.1.1 and show that $w_{n}^{1, \sigma}$ in increasing and changes sign in that interval. First, we calculate $\left(w_{n}^{1, \sigma}\right)^{\prime}(\chi)=D_{n} \mathrm{Bi}^{\prime}(\chi)-\mathrm{Ai}^{\prime}(\chi)>0$, where we have used that $\operatorname{Bi}^{\prime}(\chi)>0, \operatorname{Ai}^{\prime}(\chi)<0$, and $D_{n}>0$. Also, $w_{n}^{1, \sigma}(0)<0$ (by $\operatorname{Ai}(0)>0$ and (5.4)) and, working as in Section 5.1.1,

$$
w_{n}^{1, \sigma}\left(\gamma^{-1}+\bar{\chi}_{n}\right)=\beta^{-1} \sqrt{\gamma}\left[D_{n} \operatorname{Bi}^{\prime}\left(\gamma^{-1}+\bar{\chi}_{n}\right)-\operatorname{Ai}^{\prime}\left(\gamma^{-1}+\bar{\chi}_{n}\right)\right]>0 .
$$

This completes the proof.

5.2. The case $0<\beta<1$. In this section, we select $D_{A}$ and $D_{B}$ so that (5.1) becomes

$$
w_{n}^{1, \sigma}(\chi)=\operatorname{Ai}(\chi)+D_{n} \operatorname{Bi}(\chi), \quad \text { with } D_{n}=-\frac{\Gamma_{L, n}(\mathrm{Ai})}{\Gamma_{L, n}(\mathrm{Bi})}=-\frac{\Gamma_{R, n}(\mathrm{Ai})}{\Gamma_{R, n}(\mathrm{Bi})}
$$

Using this formula, we prove Lemma 5.1 and verify that the profile of $\omega_{0}$ has a spike around the point $x_{\beta}=\beta^{2}$.

We shall show that the eigenfunction $w_{n}^{1, \sigma}(n=0, \ldots, N)$ has $n$ zeroes in the interval $\left[\bar{\chi}_{n}, \gamma^{-1}+\right.$ $\left.\bar{\chi}_{n}\right]$. The proof is entirely analogous to that in Section 5.1.2. Here also, the $n$-th eigenvalue is $\mu_{n}^{1, \sigma}=-\gamma \sigma \bar{\chi}_{n}$, while the corresponding eigenfunction $w_{n}^{1, \sigma}$ is given by (5.5). The constant $D_{n}$ may be estimated to be

$$
D_{n}=\left(\frac{\Delta_{3}^{2}}{2}+\hat{C}_{n}(\gamma)\right) \exp \left(-\frac{4}{3 \gamma^{3 / 2}}+2 \frac{\left|A_{n+1}\right|}{\sqrt{\gamma}}-\frac{2}{\beta}\right)
$$

where $\Delta_{3}^{2}=(1+\beta) /(1-\beta)$ and $\left|\hat{C}_{n}\right|<c_{n}^{\prime} \gamma^{1 / 2}$, for some $c_{n}^{\prime}>0$. This is an estimate of the same type as (5.3) but with $A_{n+1}$ replacing $A_{n}$. Thus, the estimate (5.4) holds here as well with the 


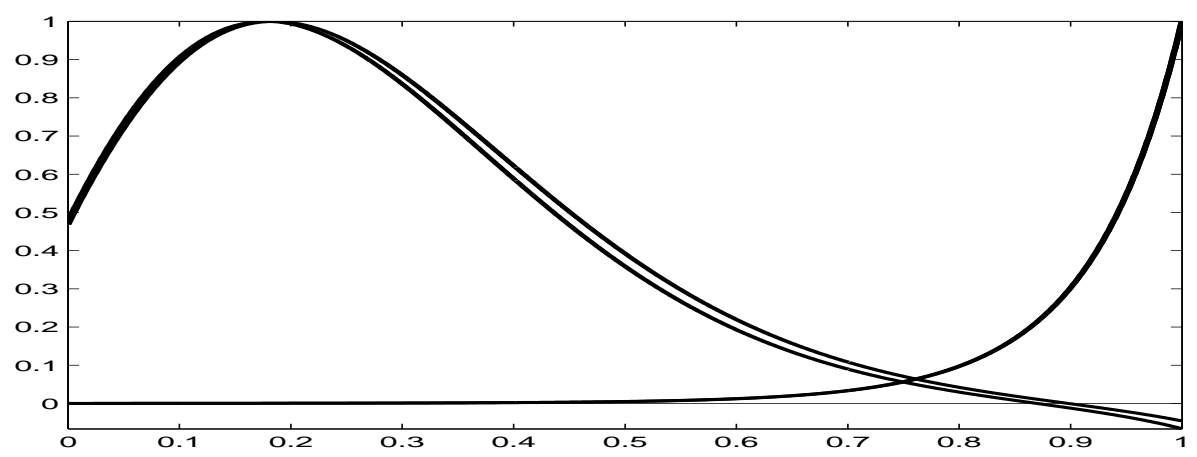

FIG. 5.1. The eigenfunctions $w_{0}^{1, \sigma_{L}}, w_{0}^{1, \sigma_{U}}$ (always positive and coinciding within plotting accuracy) and $w_{1}^{1, \sigma_{L}}$, $w_{1}^{1, \sigma_{U}}$ (changing sign). Here, $a=0.775, n_{H}=0.667, \varepsilon=0.001, \kappa=1, \ell=0.25$, and $j_{H}=0.5$, which yields $\sigma_{L}=0.1333, \sigma_{U}=0.1457$ (and thus $\sigma_{L}<\sigma_{U}<a^{2} / 4$ ), $0.0104 \leq \lambda_{0} \leq 0.0222$, and $-0.0541 \leq \lambda_{1} \leq-0.0512$. Note that $\lambda_{1}<\lambda_{0}$ and that none of $w_{0}^{1, \sigma_{L}}$ and $w_{0}^{1, \sigma_{U}}$ has zeroes in $[0,1]$, while $w_{1}^{1, \sigma_{L}}$ and $w_{1}^{1, \sigma_{U}}$ have exactly one zero in the same interval.

same change. Recalling that $\bar{\chi}_{n}$ is algebraically larger than $A_{n+1}$ (see Lemmas 4.4 and A.2), we conclude that $w_{n}^{1, \sigma}$ has $n$ distinct zeroes each of which is exponentially close to one of $A_{1}, \ldots, A_{n}$. Next, we show that $w_{n}^{1, \sigma}>0$ in $\left[0, \gamma^{-1}+\bar{\chi}_{n}\right]$ and thus has no extra zeroes. We calculate $w_{n}^{1, \sigma}(\chi)=$ $\operatorname{Ai}(\chi)+D_{n} \operatorname{Bi}(\chi)$. Now, $\operatorname{Bi}(\chi)>0$ and $\operatorname{Ai}(\chi)>0$, for all $\chi \in\left[0, \gamma^{-1}+\bar{\chi}_{n}\right]$, while $D_{n}>0$ by $(5.6)$. Hence, $w_{n}^{1, \sigma}>0$ and the proof is complete.

Next, we examine the profile of the solution $\omega_{0}$ associated with $w_{0}$. Working as in Section 5.1.1, we calculate

$$
\omega_{0}(x)=C_{I I}(x) x^{-1 / 4} \exp \left(\frac{\beta}{\gamma^{3 / 2}} x\right) \cosh \left(\theta_{2}(x)\right), \quad x \in[0,1]
$$

where $C_{I I}(x)=C_{I I, 0}+C_{I I, 1}(x), \sup _{[0,1]}\left|C_{I I, 1}(x)\right|<c_{I I} \sqrt{\gamma}$ for some $c_{I I}>0$, and

$$
\theta_{2}(x)=\frac{2}{3 \gamma^{3 / 2}}\left(1-x^{3 / 2}\right)-\left(\frac{\left|A_{1}\right|}{\sqrt{\gamma}}-\frac{1}{\beta}\right)(1-\sqrt{x})-\log \Delta_{3} .
$$

The first two terms in the right member of the expression for $\omega_{0}$ are bounded, while the other two correspond to boundary layers at $x=1$ and $x=0$, respectively. A straightforward calculation shows that $\omega_{0}$ corresponds to a spike around the point $x_{\beta}$, where

$$
\left|x_{\beta}-\left(\beta^{2}+\left|A_{1}\right| \gamma\right)\right|<c \gamma^{2}, \quad \text { for some } \quad c>0
$$

We remark here that $x_{\beta}$ does not correspond to the position of the DCM. This information is obtained in the next section, instead, through a WKB analysis.

6. The WKB approximation. In the previous sections, we derived strict bounds for the eigenvalues $\mu_{1}, \ldots, \mu_{N}$ of $\mathcal{L}$ and summarized them in Theorem 2.1. In this section, we use the WKB method to derive explicit (albeit asymptotic) formulas for these eigenvalues. The outcome of this analysis has already been summarized in Section 2 .

6.1. The case $a^{2} / 4<\sigma_{L}$. 
6.1.1. WKB formulas for $w$. The eigenvalue problem (3.2) reads

$$
\varepsilon w_{x x}=(F(x)-\mu) w, \quad \text { with } \quad \mathcal{G}(w, 0)=\mathcal{G}(w, 1)=0 .
$$

Since we are interested in the regime $\sigma_{L}>a^{2} / 4$, Lemma 3.3 states that the eigenvalues $\mu_{0}, \ldots, \mu_{N}$ lie in a $\mathcal{O}\left(\varepsilon^{1 / 3}\right)$ region to the right of zero. Thus, for any $0 \leq n \leq N$,

$$
F(x)<\mu_{n}, \quad \text { for } x \in\left[0, \bar{x}_{n}\right), \quad \text { and } \quad F(x)>\mu_{n}, \quad \text { for } x \in\left(\bar{x}_{n}, 1\right] .
$$

Here, $\bar{x}_{n}$ corresponds to a turning point, i.e., $F\left(\bar{x}_{n}\right)=\mu_{n}$, and it is given by the formula

$$
\bar{x}_{n}=\frac{1}{\kappa} \log \frac{1+\mu_{n}\left(1+\eta_{H}\right)\left(1+j_{H}^{-1}\right)}{1-\mu_{n}\left(1+\eta_{H}\right)\left(1+j_{H}\right)} .
$$

Lemmas 3.3 and A.2 suggest that the eigenvalue $\mu_{n}$ may be expanded asymptotically in powers of $\varepsilon^{1 / 6}$ starting with $\mathcal{O}\left(\varepsilon^{1 / 3}\right)$ terms, $\mu_{n}=\sum_{\ell=2}^{\infty} \varepsilon^{\ell / 6} \mu_{n, \ell}$. Thus, we also find

$$
\bar{x}_{n}=\varepsilon^{1 / 3} \sigma_{0}^{-1} \mu_{n, 2}+\varepsilon^{1 / 2} \sigma_{0}^{-1} \mu_{n, 3}+\mathcal{O}\left(\varepsilon^{2 / 3}\right), \quad \text { where } \sigma_{0}=F^{\prime}(0) .
$$

The solution in the region $\left(\bar{x}_{n}, 1\right]$, where $F(x)-\mu_{n}>0$, can be determined using standard formulas ([2, Ch. 10, Sect. 1]),

$$
w_{n}(x)=\left[F(x)-\mu_{n}\right]^{-1 / 4}\left[C_{a} \mathrm{e}^{-\int_{\bar{x}_{n}}^{x} \sqrt{\left(F(s)-\mu_{n}\right) / \varepsilon} d s}+C_{b} \mathrm{e}^{\int_{\bar{x}_{n}}^{x} \sqrt{\left(F(s)-\mu_{n}\right) / \varepsilon} d s}\right] .
$$

Here, $C_{a}$ and $C_{b}$ are arbitrary constants, to leading order in $\varepsilon$. (Higher-order terms in the asymptotic expansions of $C_{a}$ and $C_{b}$ generally depend on $x$, see [2] for details.) Using this information and the asymptotic expansion for $\mu_{n}$, we may determine the principal part of the solution $w_{n}$,

$$
w_{n, 0}(x)=[F(x)]^{-1 / 4}\left[C_{a, 0} \mathrm{e}^{-\theta_{3}(x)}+C_{b, 0} \mathrm{e}^{\theta_{3}(x)}\right],
$$

for arbitrary constants $C_{a, 0}$ and $C_{b, 0}$ and where

$$
\theta_{3}(x)=\frac{1}{\varepsilon^{1 / 2}} \int_{0}^{x} \sqrt{F(s)} d s-\frac{1}{\varepsilon^{1 / 6}} \frac{\mu_{n, 2}}{2} \int_{0}^{x} \frac{d s}{\sqrt{F(s)}}+\frac{\mu_{n, 2}}{\sqrt{\sigma_{0}}}-\frac{2}{3} \sqrt{\sigma_{0}}-\frac{\mu_{n, 3}}{2} \int_{0}^{x} \frac{d s}{\sqrt{F(s)}}
$$

To determine the solution in $\left[0, \bar{x}_{n}\right)$, we change independent variable through

$$
x=\varepsilon^{1 / 3} \sigma_{0}^{-1 / 3}\left(\chi-\bar{\chi}_{n}\right), \quad \text { where } \quad \bar{\chi}_{n}=-\sigma_{0}^{1 / 3} \varepsilon^{-1 / 3} \bar{x}_{n}=-\sigma_{0}^{-2 / 3} \mu_{n, 2}+\mathcal{O}\left(\varepsilon^{1 / 2}\right)<0
$$

and expand $F(x)-\mu_{n}$ asymptotically,

$$
F(x)-\mu_{n}=F\left(\varepsilon^{1 / 3} \sigma_{0}^{-1 / 3}\left(\chi-\bar{\chi}_{n}\right)\right)-\mu_{n}=\varepsilon^{1 / 3} \sigma_{0}^{2 / 3} \chi+\mathcal{O}\left(\varepsilon^{1 / 2}\right) .
$$

As a result, $(6.1)$ becomes, to leading order, the Airy equation $\left(w_{n}\right)_{\chi \chi}=\chi w_{n}$, whence

$$
w_{n, 0}(\chi)=D_{a, 0} \operatorname{Ai}(\chi)+D_{b, 0} \operatorname{Bi}(\chi), \quad \text { with } \chi \in\left(-\sigma_{0}^{-2 / 3} \mu_{n, 2}, 0\right]
$$

6.1.2. Boundary conditions for the WKB solution. Next, we determine the coefficients appearing in (6.5) and (6.9). Formula (6.5) represents the solution in the region $\left(\bar{x}_{n}, 1\right]$, and thus it must satisfy the boundary condition at $x=1, \mathcal{G}\left(w_{n}, 1\right)=0$. Using (3.3), we find, to leading order,

$$
C_{a, 0}\left(a+2 \sqrt{\sigma_{1}}\right) \mathrm{e}^{-\theta_{3}(x)}+C_{b, 0}\left(a-2 \sqrt{\sigma_{1}}\right) \mathrm{e}^{\theta_{3}(x)}=0, \quad \text { where } \sigma_{1}=F(1) .
$$


Next, the formula given in (6.9) is valid for $\chi \in\left(-\sigma_{0}^{-2 / 3} \mu_{n, 2}, 0\right]$ (equivalently, for $x \in\left[0, \bar{x}_{n}\right)$ ), and thus it must satisfy the boundary condition $\mathcal{G}(w, 0)=0$. Recasting the formula for $\mathcal{G}$ given in $(3.3)$ in terms of $\chi$, we obtain to leading order

$$
D_{a, 0} \mathrm{Ai}\left(-\sigma_{0}^{-2 / 3} \mu_{n, 2}\right)+D_{b, 0} \mathrm{Bi}\left(-\sigma_{0}^{-2 / 3} \mu_{n, 2}\right)=0 .
$$

Finally, (6.5) and (6.9) should also match in an intermediate length scale to the right of $x=\bar{x}_{n}$ (equivalently, of $\chi=0$ ). To this end, we set $\psi=\varepsilon^{d}\left(x-\bar{x}_{n}\right)$, where $1 / 5<d<1 / 3$ [2, Sect. 10.4], and recast (6.5) in terms of $\psi$. We find, to leading order and for all $\mathcal{O}(1)$, positive values of $\psi$,

$$
w_{n, 0}(x(\psi))=\varepsilon^{-d / 4} \sigma_{0}^{-1 / 4} \psi^{-1 / 4}\left[C_{a, 0} \mathrm{e}^{-\theta_{4}(\psi)-\sigma_{0}^{-1}\left(\mu_{n, 2}\right)^{3 / 2}}+C_{b, 0} \mathrm{e}^{\theta_{4}(\psi)+\sigma_{0}^{-1}\left(\mu_{n, 2}\right)^{3 / 2}}\right],
$$

where $\theta_{4}(\psi)=(2 / 3) \varepsilon^{(3 d-1) / 2} \sqrt{\sigma_{0}} \psi^{3 / 2}$. Similarly, (6.9) yields

$$
w_{n, 0}(\chi(\psi))=\varepsilon^{1 / 12-d / 4} \sigma_{0}^{-1 / 12} \pi^{-1 / 2} \psi^{-1 / 4}\left[\frac{D_{a, 0}}{2} \mathrm{e}^{-\theta_{4}(\psi)}+D_{b, 0} \mathrm{e}^{\theta_{4}(\psi)}\right] .
$$

The matching condition around the turning point gives, then,

$$
C_{a, 0}=\varepsilon^{1 / 12} \frac{\sigma_{0}^{1 / 6}}{2 \sqrt{\pi}} \mathrm{e}^{\sigma_{0}^{-1}\left(\mu_{n, 2}\right)^{3 / 2}} D_{a, 0} \quad \text { and } \quad C_{b, 0}=\varepsilon^{1 / 12} \frac{\sigma_{0}^{1 / 6}}{\sqrt{\pi}} \mathrm{e}^{-\sigma_{0}^{-1}\left(\mu_{n, 2}\right)^{3 / 2}} D_{b, 0}
$$

6.1.3. The eigenvalues $\mu_{0}, \ldots, \mu_{n}$. The linear system (6.10)-(6.12) has a nontrivial solution if and only if the determinant corresponding to it vanishes identically,

$$
2\left(a-2 \sqrt{\sigma_{1}}\right) \mathrm{e}^{\theta_{3}(1)-\sigma_{0}^{-1}\left(\mu_{n, 2}\right)^{3 / 2}} \operatorname{Ai}\left(\sigma^{-2 / 3} \mu_{n, 2}\right)+\left(a+2 \sqrt{\sigma_{1}}\right) \mathrm{e}^{-\theta_{3}(1)+\sigma_{0}^{-1}\left(\mu_{n, 2}\right)^{3 / 2}} \operatorname{Bi}\left(\sigma^{-2 / 3} \mu_{n, 2}\right)=0 .
$$

Since $\sigma_{1} \geq \sigma_{L}$ by Lemma 2.1 and $\sigma_{L}>a^{2} / 4$ by assumption, $a-2 \sqrt{\sigma_{1}}$ is $\mathcal{O}(1)$ and negative. Also, $\theta_{3}(1)$ is $\mathcal{O}(1)$ and positive by (6.6). Thus, the determinant condition reduces to $\operatorname{Ai}\left(\sigma^{-2 / 3} \mu_{n, 2}\right)=0$, whence $\mu_{n, 2}=-\sigma_{0}^{2 / 3} A_{n}=\sigma_{0}^{2 / 3}\left|A_{n}\right|>0$. Hence, we find for the eigenvalues of (1.13),

$$
\lambda_{n}=\lambda^{*}-\varepsilon^{1 / 3} \sigma_{0}^{2 / 3}\left|A_{n}\right|+\mathcal{O}\left(\varepsilon^{1 / 2}\right) .
$$

Working in a similar way, we find $\mu_{n, 3}=-2 \sigma_{0} / a$.

Recalling that $\sigma_{0}=F^{\prime}(0)=-f^{\prime}(0)$ by (2.1) and Lemma 2.1 (see also Fig. 2.3), we find that the WKB formula (6.13) coincides - up to and including terms of $\mathcal{O}(1)$ and $\mathcal{O}\left(\varepsilon^{1 / 3}\right)$ - (a) for $0<j_{H}<j_{H}^{(2)}$, with the rigorous lower bound for $\lambda_{n}$ derived in Theorem 2.1 and (b) for $j_{H}>1$, with the rigorous upper bound for $\lambda_{n}$ derived in the same theorem. For the remaining values of $j_{H},(6.13)$ yields a value for $\lambda_{n}$ which lies in between the rigorous bounds derived in Theorem 2.1-indeed, in that case, $\sigma_{L}<F^{\prime}(0)<\sigma_{U}$, see Fig. 2.3.

6.1.4. The eigenfunctions $w_{0}, \ldots, w_{n}$. Finally, one may determine the constants $C_{a}, C_{b}, D_{a}$, and $D_{b}$ corresponding to the eigenfunction $w_{n}$, and thus also $w_{n}$ itself, through (6.10)-(6.12). The principal part of $w_{n}$ is given by the formula

$$
w_{n, 0}(x)= \begin{cases}\operatorname{Ai}\left(A_{n}+\varepsilon^{-1 / 3} \sigma_{0}^{1 / 3} x\right), & \text { for } x \in\left[0, \varepsilon^{1 / 3} \sigma_{0}^{-1 / 3}\left|A_{n}\right|\right), \\ C[F(x)]^{-1 / 4} \cosh \Theta(x), & \text { for } x \in\left(\varepsilon^{1 / 3} \sigma_{0}^{-1 / 3}\left|A_{n}\right|, 1\right] .\end{cases}
$$

Here,

$$
\begin{gathered}
C=\varepsilon^{1 / 12} \frac{\sigma_{0}^{1 / 6}}{2 \sqrt{\pi}} \Delta_{4} \mathrm{e}^{\left|A_{n}\right|^{3 / 2}-\theta_{3}(1)}, \quad \text { where } \Delta_{4}^{2}=\frac{2 \sqrt{\sigma_{1}}+a}{2 \sqrt{\sigma_{1}}-a} \\
\Theta(x)=\varepsilon^{-1 / 2} \int_{x}^{1} \sqrt{F(s)} d s-\left(\varepsilon^{-1 / 6} \frac{\sigma_{0}^{2 / 3}\left|A_{n}\right|}{2}-\frac{\sigma_{0}}{a}\right) \int_{x}^{1} \frac{d s}{\sqrt{F(s)}}+\log \Delta_{4} .
\end{gathered}
$$




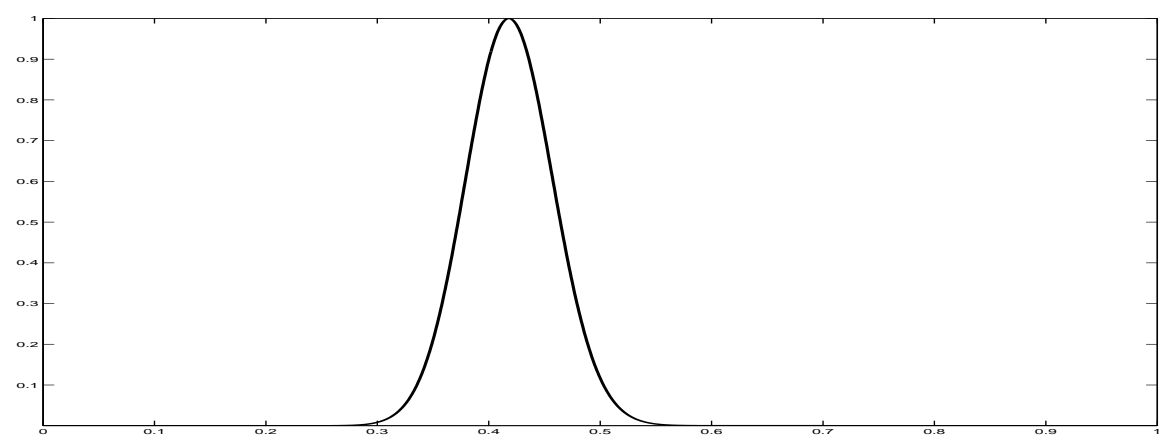

FIG. 6.1. The eigenfunction $\omega_{0}$ as given by (6.14). Here, $a=0.5, n_{H}=0.667, \varepsilon=2 \cdot 10^{-7}, \kappa=1$, and $j_{H}=0.5$. The eigenfunction has been scaled so that its maximum value is equal to one.

Recalling (3.1), we find

$$
\omega_{n, 0}(x)= \begin{cases}\mathrm{e}^{a x / 2 \sqrt{\varepsilon}} \mathrm{Ai}\left(A_{n}+\varepsilon^{-1 / 3} \sigma_{0}^{1 / 3} x\right), & \text { for } x \in\left[0, \varepsilon^{1 / 3} \sigma_{0}^{-1 / 3}\left|A_{n}\right|\right), \\ C[F(x)]^{-1 / 4} \mathrm{e}^{a x / 2 \sqrt{\varepsilon}} \cosh \Theta(x), & \text { for } x \in\left(\varepsilon^{1 / 3} \sigma_{0}^{-1 / 3}\left|A_{n}\right|, 1\right] .\end{cases}
$$

A straightforward calculation shows that $\omega_{0}$ corresponds to a spike around the point

$$
x_{\mathrm{DCM}}=x_{\mathrm{DCM}, 0}+\mathcal{O}\left(\varepsilon^{1 / 3}\right),
$$

where $x_{\mathrm{DCM}, 0}$ is the unique solution to $F\left(x_{\mathrm{DCM}, 0}\right)=a^{2} / 4$. Thus, $\omega_{0,0}$ indeed corresponds to a DCM. Furthermore, the location of the maximum phytoplankton concentration is expressed explicitly by this equation in terms of the rescaled biological parameters $\kappa, \eta_{H}, j_{H}$, and $a$.

6.2. The case $a^{2} / 4>\sigma_{U}$. To obtain the eigenvalues and their corresponding eigenfunctions in this case, we work as in the preceding section. Here also, the eigenvalue problem (3.2) has the form (6.1). Since $a^{2} / 4>\sigma_{U}$, the eigenvalue $\mu_{0}$ is $\mathcal{O}(1)$ and negative, while $\mu_{1}, \ldots, \mu_{N}$ are $\mathcal{O}\left(\varepsilon^{1 / 3}\right)$ and positive, see Lemma 3.3. Due to the qualitative difference between $\mu_{0}$ and the eigenvalues of higher order, we consider them separately.

We start with the case $1 \leq n \leq N$. Then, for each such $n$, the eigenvalue problem (6.1) has a unique turning point $\bar{x}_{n}$ given by (6.2), and the analysis presented in the preceding section applies here also. The formulas for $\mu_{n}$ and $\omega_{n}, 1 \leq n \leq N$, are identical to those of the preceding section, with the sole modification that $A_{n}$ in $(6.13)-(6.16)$ must be replaced by $A_{n-1}$. This completes the analysis for the case $1 \leq n \leq N$.

Next, we treat the case $n=0$. Since $\mu_{0}<0<F(x)$ for all $x \in[0,1]$, the eigenvalue problem (6.1) corresponding to $\mu_{0}$ has no turning points. Thus, the WKB formula (6.4), with $n=0$ and $\bar{x}_{n}$ replaced by zero, is valid for all $x \in[0,1]$. Lemmas 3.3 and A.2 suggest that $\mu_{0}$ may be expanded asymptotically as $\mu_{0}=\sum_{\ell=0}^{\infty} \varepsilon^{\ell / 2} \mu_{0, \ell}$. Using this expansion, we calculate the principal part of $w_{0}$,

$$
w_{0,0}(x)=\left[F(x)-\mu_{0,0}\right]^{-1 / 4}\left[C_{a, 0} \mathrm{e}^{-\theta_{5}(x)}+C_{b, 0} \mathrm{e}^{\theta_{5}(x)}\right],
$$

where $C_{a, 0}$ and $C_{b, 0}$ are arbitrary constants and

$$
\theta_{5}(x)=\frac{1}{\varepsilon^{1 / 2}} \int_{0}^{x} \sqrt{F(s)-\mu_{0,0}} d s-\frac{\mu_{0,1}}{2} \int_{0}^{x} \frac{d s}{\sqrt{F(s)-\mu_{0,0}}} .
$$


Next, recalling the boundary conditions $\mathcal{G}(w, 0)=\mathcal{G}(w, 1)=0$, we obtain, to leading order,

$$
\begin{array}{r}
C_{a, 0}\left(a+2 \sqrt{-\mu_{0,0}}\right)+C_{b, 0}\left(a-2 \sqrt{-\mu_{0,0}}\right)=0 \\
C_{a, 0}\left(a+2 \sqrt{\sigma_{1}-\mu_{0,0}}\right) \mathrm{e}^{-\theta_{5}(1)}+C_{b, 0}\left(a-2 \sqrt{\sigma_{1}-\mu_{0,0}}\right) \mathrm{e}^{\theta_{5}(1)}=0
\end{array}
$$

where we recall that $\sigma_{1}=F(1)$. Here, $\theta_{5}(1)$ is $\mathcal{O}(1)$ and positive by $(6.20)$, while $a+2 \sqrt{-\mu_{0,0}}>0$. Thus, we find, to leading order, $\mu_{0,0}=F(1)-a^{2} / 4$, whence

$$
\lambda_{0,0}=f(1)-\ell .
$$

Using this formula, one may also determine $C_{a, 0}$ and $C_{b, 0}$ to obtain $w_{0,0}$,

$$
w_{0,0}(x)=\left[F(x)-\mu_{0,0}\right]^{-1 / 4} \sinh \Phi(x),
$$

for $x \in[0,1]$ and up to a multiplicative constant. Here,

$$
\Phi(x)=\frac{1}{\varepsilon^{1 / 2}} \int_{0}^{x} \sqrt{F(s)-\mu_{0,0}} d s-\frac{\mu_{0,1}}{2} \int_{0}^{x} \frac{d s}{\sqrt{F(s)-\mu_{0,0}}}+\log \Delta_{5},
$$

where

$$
\Delta_{5}^{2}=\frac{\beta_{1}+\sqrt{\beta_{1}^{2}-1}}{\beta_{1}-\sqrt{\beta_{1}^{2}-1}}, \quad \beta_{1}=\frac{a}{2 F(1)} .
$$

Recalling (3.1), we find

$$
\omega_{0,0}(x)=\left[F(x)-\mu_{0,0}\right]^{-1 / 4} \mathrm{e}^{a x / 2 \sqrt{\varepsilon}} \sinh \Phi(x), \quad \text { for } x \in[0,1] .
$$

The profile of $\omega_{0}$ corresponds to a boundary layer at the point $x=1$.

6.3. The transitional regime $\sigma_{L}<a^{2} / 4<\sigma_{U}$. Equations (6.13) and (6.22) may be used to derive information for the transitional regime $\sigma_{L}<a^{2} / 4<\sigma_{U}$ (see Theorem 2.1 and the discussion in Section 2). In particular, the transition between the case where $\lambda_{0}$ is associated with a boundary layer (in biological terms, with a BL) and the case where it is associated with a spike (that is, with a DCM) occurs, to leading order, when $f(1)-\ell=\lambda^{*}$. Recalling (2.4), we rewrite this equation as

$$
F(1)=f(0)-f(1)=a^{2} / 4 \text {. }
$$

As we mentioned in Section 2, this condition reduces, to leading order, to $a^{2} / 4=\sigma_{U}$, for $0<j_{H} \leq$ $j_{H}^{(1)}$, and to $a^{2} / 4=\sigma_{L}$, for $j_{H} \geq j_{H}^{(2)}$. For $j_{H}^{(1)}<j_{H}<j_{H}^{(2)}$, this transitional value of $a^{2} / 4$ falls between $\sigma_{U}$ and $\sigma_{L}$, see Fig. 2.3.

\section{Bifurcations and simulations.}

7.1. The bifurcation diagram . In this section, we use the WKB expressions for the first few eigenvalues derived in Section 6 to identify the bifurcations that system (3.2) undergoes. In this way, we identify the regions in the parameter space where the BL and DCM steady states become stable. As already mentioned in Section 1, we are primarily interested in the effect of environmental conditions - in particular, of nutrient concentration and diffusion - on phytoplankton. For this reason, we choose to vary the parameters $\eta_{H}=N_{H} / N_{B}$ (which encapsulates information pertaining to the nutrient levels and nutrient absorption by phytoplankton) and $a=V / \sqrt{\mu D}$ (which is a measure of diffusion) (see (1.7)). The remaining four dimensionless parameters $\left(\varepsilon, \kappa, j_{H}\right.$, and $\ell)$ are kept constant. For simplicity of presentation, we define the variables

$$
\nu=\left(1+\eta_{H}\right)^{-1} \text { and } A=a^{2} / 4 .
$$


The curves separating the regions in the $(\nu, A)$ - plane which are characterized by qualitatively different behavior of the rescaled model (1.5), (1.8) may be found by recasting (6.13) and (6.22) in terms of the rescaled parameters. In particular, using (1.12), (2.1), and (2.4), we find (see Fig. 7.1) - In regions $I$ and $I I, \lambda_{0}$ is given by (6.22) (in region $I$ ) and by (6.13) with $n=0$ (in region $I I$ ). In either case, $\lambda_{0}<0$, and hence the zero (trivial) state is stable.

- In region $I I I, \lambda_{0}$ is given by (6.13) and it is positive. In fact, the further into this region one goes, the more eigenvalues cross zero and become positive, since they are $\mathcal{O}\left(\varepsilon^{1 / 3}\right)$ apart by (6.13). All of these eigenvalues are associated with DCMs.

- In region $V I, \lambda_{0}$ is given by $(6.22)$ and it is positive, while all other eigenvalues are negative. Thus, the only bifurcating patterns in this regime are BL profiles.

- Finally, in regions $I V$ and $V$, eigenvalues associated with both BB and DCM profiles are positive, and thus no further info can be derived from our linear analysis.

The boundaries of these regions may be deduced explicitly in the aforementioned manner. First, setting the expression for $\lambda_{0}$ in (6.22) equal to zero, we obtain, to leading order, the vertical line separating the regions $I, I I$, and $I I I$ from the regions $I V, V$, and $V I$,

$$
\nu=\ell\left(1+\mathrm{e}^{\kappa} j_{H}\right)
$$

Next, setting the expression for $\lambda_{0}$ in $(6.13)$ equal to zero, we obtain, to leading order, the diagonal line separating the regions $I, I I$, and $V I$ from $I I I, I V$, and $V$,

$$
A=\frac{1}{1+j_{H}} \nu-\ell
$$

Finally, setting the expressions for $\lambda_{0}$ in (6.13) and (6.22) equal to each other, we obtain the transitional regime (6.24). In terms of the rescaled parameters, we find

$$
A=\left(\frac{1}{1+j_{H}}-\frac{1}{1+\mathrm{e}^{\kappa} j_{H}}\right) \nu .
$$

Since the physical region $n_{H}>0$ corresponds to the region $0<\nu<1$, the formulas above imply that (a) for $0<\ell<\left(1+\mathrm{e}^{\kappa} j_{H}\right)^{-1}$, both a BL and a DCM may bifurcate, (b) for $\left(1+\mathrm{e}^{\kappa} j_{H}\right)^{-1}<\ell<$ $\left(1+j_{H}\right)^{-1}$, only a DCM may bifurcate, and (c) for $\ell>\left(1+j_{H}\right)^{-1}$, the trivial state is stable.

REMARK 7.1. Similar information may be derived by the rigorous bounds in Theorem 2.1, with the important difference that the divided curves have to be replaced by regions of finite thickness.

7.2. Numerical simulations . In this section, we present numerical simulations on the full model (1.1)-(1.4), and we compare the results with our theoretical predictions. The parameters are chosen in biologically relevant regions [11].

We considered first the validity of our asymptotic analysis, i.e., we checked whether the analytically obtained bounds for the occurrence of the DCMs and BLs - see Theorem 2.1, Section 7.1, Fig. 7.1, and Remark 7.1 - can be recovered by numerical simulations of the PDE (1.1)-(1.4). We used the numerical method described in Remark 7.2 at each node of a two-dimensional grid of a part of the $(\nu, A)$ parameter plane (keeping all other parameters fixed) to determine the attracting pattern generated by (1.1)-(1.8) and chose the initial profile at each node in the parameter space to be the numerically converged pattern for an adjacent node at the previous step.

In Figure 7.2, we present the region near the co-dimension 2 point in the $(\nu, A)$ parameter plane at which both the DCMs and the BLs bifurcate (with all other parameters fixed: $\varepsilon=9 \cdot 10^{-5}, \ell=0.2$, $\left.j_{H}=0.5, \kappa=1\right)$. Away from this co-dimension 2 point, the numerically determined bifurcation curves are clearly within the bounds given by Theorem 2.1 and thus confirm our analysis. Note that 


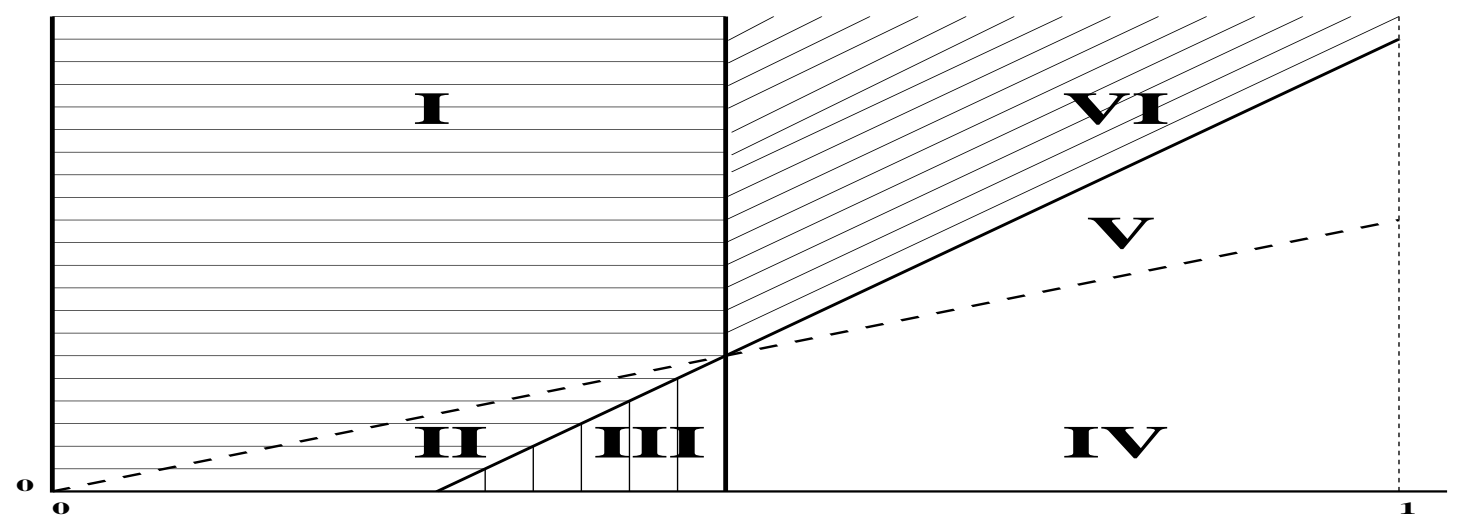

FIG. 7.1. The bifurcation diagram in the $(\nu, A)$-plane. The horizontal axis corresponds to $\nu=\left(1+\eta_{H}\right)^{-1}$, while the vertical one to $A=a^{2} / 4$. In the region shaded horizontally, the trivial, zero state is stable. In the region shaded vertically, DCMs bifurcate, while BL profiles remain damped. In the region shaded diagonally, BL profiles bifurcate, while DCM profiles remain damped. Finally, in the unshaded region, both profiles grow linearly.

this implies that the bifurcations have a supercritical nature - an observation that does not follow from our linear analysis. Near the co-dimension 2 point, there seems to be a slight discrepancy between our analysis and the numerical findings. First, we note that the bifurcation from the trivial state (with no phytoplankton) to the blooming DCM state is not exactly in the region determined by Theorem 2.1. However, for this combination of parameters, this region is quite narrow - which indicates the accuracy of our analysis. In fact, it is more narrow than the width of the rectangular grid of the $(\nu, A)$ parameter plane we used to determine Figure 7.2, which implies that the simulations do agree with the analysis. The other discrepancy, namely the occurrence of a small 'triangle' of BL patterns in the region where one would expect DCMs, is clearly related to the presence of the co-dimension 2 point. To understand the true nature of the dynamics, one needs to perform a weakly nonlinear analysis near this point, and, most probably, a more detailed numerical analysis that distinguishes between DCMs, BLs and patterns that have the structure of a combined DCM and BL. This is the topic of work in progress.

Unlike the simulations presented in [11], we only briefly considered the secondary bifurcations here. Figure 7.3 exhibits the primary bifurcation of the trivial state into a DCM, and the secondary bifurcation (of Hopf type) of the DCM into an oscillating DCM (see [11] for more (biological) details on this behavior). A priori, one would guess that this Hopf bifurcation cannot be covered by our linear stability analysis of the trivial state. However, in Figure 7.3 we also plotted the leading order approximations of the curves at which the first two eigenvalues associated to the stability of the trivial state, $\lambda_{0}$ and $\lambda_{1}$, cross through the imaginary axis. It follows that the distance (in parameter space) between the primary and the secondary bifurcations is of the same order, $\mathcal{O}\left(\varepsilon^{1 / 3}\right)$, as the distance between the successive eigenvalues $\lambda_{n}$. This is crucial information for the subsequent (weakly) nonlinear analysis, since the fact that the DCM undergoes its secondary Hopf bifurcation for parameter combinations that are asymptotically close (in $\varepsilon$ ) to the primary bifurcation implies that the above a priori guess is not correct and that the stability and bifurcation analysis of the DCM can indeed be based on the linear analysis presented in the preceding sections. The higher order eigenvalues $\lambda_{1}, \lambda_{2}, \ldots$, the associated eigenfunctions $\omega_{1}(x), \omega_{2}(x), \ldots$ and their 'slaved' $\eta$-components $\eta_{1}(x), \eta_{2}(x), \ldots$ (which are not considered here but can be determined explicitly through (1.11)), will serve as necessary input for this nonlinear analysis.

Thus, a 'full' linear stability analysis of the uncoupled system (1.13) as presented here may serve as a foundation for the analysis of secondary bifurcations that can only occur in the coupled system (see Section 1 and [14]). This feature is very special and quite uncommon in explicit models. It is 


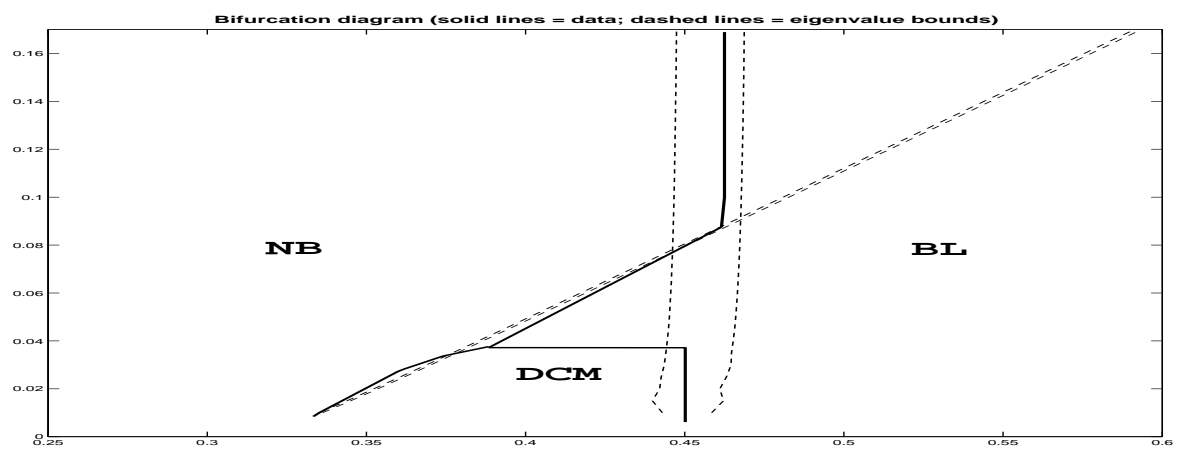

Fig. 7.2. The bifurcation diagram in the $(\nu, A)$-plane for $\varepsilon=9 \cdot 10^{-5}, \ell=0.2, j_{H}=0.5, \kappa=1$. ('NB' stands for 'No Blooming.') The solid curves correspond to numerical simulations, while the dashed ones correspond to the bounds predicted theoretically, see Theorem 2.1.

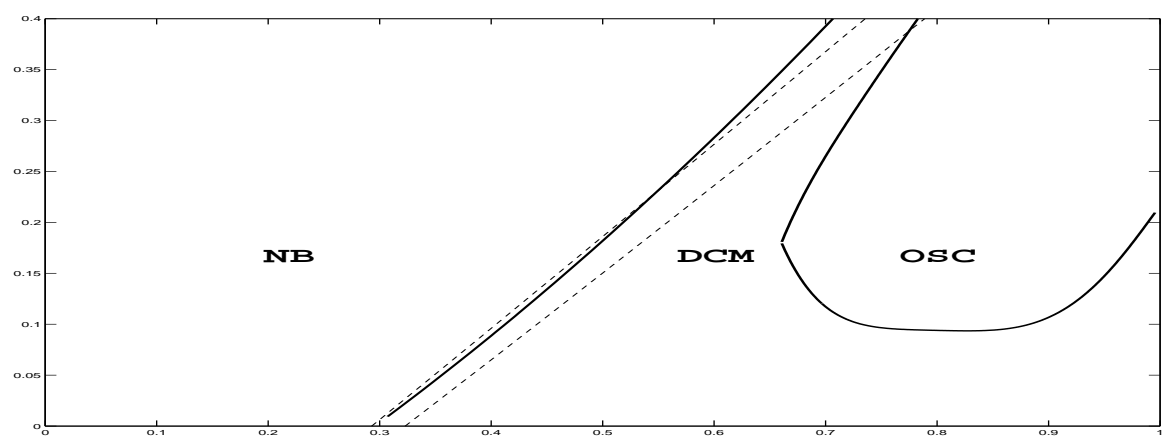

FIG. 7.3. The bifurcation diagram in the $(\nu, A)$-plane for $\varepsilon=9 \cdot 10^{-5}, \ell=0.25, j_{H}=0.033, \kappa=20$. Region $N B$ corresponds to no blooming and region OSC to oscillatory DCMs. The solid curves correspond to numerical simulations and the dashed ones to the points at which $\lambda_{0}$ (left line) and $\lambda_{1}$ (right line) cross zero, see (6.13) and Fig. 7.1. For these parameter values, the bifurcation of the BLs occurs in a non-physical part of the domain.

due to the natural singularly perturbed nature of the scaled system (1.5) - Section 1 - and it provides an opportunity to obtain fundamental insight in the dynamics of phytoplankton. This analysis, including the aforementioned co-dimension 2 analysis and the associated secondary bifurcations of BLs, is the topic of work in progress.

REMARK 7.2. The numerical results were obtained by the 'Method of Lines' approach. First, we discretized the spatial derivatives approximating the diffusion terms in the model by second-order symmetric formulae and employing a third-order upwind-biased method to discretize the advection term (see [13] for the suitability of these schemes to the current problem). Next, we integrated the resulting system of ODEs forward in time with the widely used time-integration code VODE ([3] and http://www.netlib.org/ode/). Throughout all simulations, we combined a spatial grid of a sufficiently high resolution with a high precision time integration to ensure that the conclusions drawn from the simulations are essentially free of numerical errors.

Appendix A. Basic properties of the Airy functions . In this section, we summarize some properties of the Airy functions Ai and Bi which we use repeatedly. 
Lemma A.1. Let $p>0$ and $q$ be real numbers and $\beta$ be defined as in (4.1). Then,

$$
\begin{aligned}
\Gamma\left(\mathrm{Ai}, \gamma^{-1} p+q\right)= & \left(\pi^{-1 / 2} / 2\right)\left(\gamma p^{-1}\right)^{1 / 4} \exp \left(-(2 / 3)\left(\gamma^{-1} p\right)^{3 / 2}-q\left(\gamma^{-1} p\right)^{1 / 2}\right) \\
& \cdot\left[\left(1+\beta^{-1} \sqrt{p}\right)\left(1-\left(q^{2} / 4\right)\left(\gamma p^{-1}\right)^{1 / 2}+(q / 4)\left(q^{3} / 8-1\right) \gamma p^{-1}\right)\right. \\
& \left.-(1 / 48)\left(5-5 q^{3}+q^{6} / 8-\left(43-q^{3}-q^{6} / 8\right) \beta^{-1} \sqrt{p}\right)\left(\gamma p^{-1}\right)^{3 / 2}\right], \quad \gamma \downarrow 0, \\
\Gamma\left(\mathrm{Bi}, \gamma^{-1} p+q\right)= & \pi^{-1 / 2}\left(\gamma p^{-1}\right)^{1 / 4} \exp \left((2 / 3)\left(\gamma^{-1} p\right)^{3 / 2}+q\left(\gamma^{-1} p\right)^{1 / 2}\right) \\
& {\left[\left(1-\beta^{-1} \sqrt{p}\right)\left(1+\left(q^{2} / 4\right)\left(\gamma p^{-1}\right)^{1 / 2}+(q / 4)\left(q^{3} / 8-1\right) \gamma p^{-1}\right)\right.} \\
& \left.+(1 / 48)\left(5-5 q^{3}+q^{6} / 8+\left(43-q^{3}-q^{6} / 8\right) \beta^{-1} \sqrt{p}\right)\left(\gamma p^{-1}\right)^{3 / 2}\right], \quad \gamma \downarrow 0,
\end{aligned}
$$

where the remainders of $\mathcal{O}\left(\gamma^{2}\right)$ were omitted from within the square brackets.

Proof. We only derive the first of these asymptotic expansions. The second one is derived in an entirely analogous manner. Definition (4.2) yields

$$
\Gamma\left(\mathrm{Ai}, \gamma^{-1} p+q\right)=\operatorname{Ai}\left(\gamma^{-1} p+q\right)-\beta^{-1} \sqrt{\gamma} \operatorname{Ai}^{\prime}\left(\gamma^{-1} p+q\right) .
$$

Then, we recall the standard asymptotic expansions [2]

$$
\begin{aligned}
& \operatorname{Ai}(z)=\left(\pi^{-1 / 2} z^{-1 / 4} / 2\right) \exp \left(-(2 / 3) z^{3 / 2}\right)\left[1-(5 / 48) z^{-3 / 2}+\mathcal{O}\left(z^{-3}\right)\right], \quad z \uparrow \infty \\
& \operatorname{Ai}^{\prime}(z)=-\left(\pi^{-1 / 2} z^{1 / 4} / 2\right) \exp \left(-(2 / 3) z^{3 / 2}\right)\left[1+(7 / 48) z^{-3 / 2}+\mathcal{O}\left(z^{-3}\right)\right], \quad z \uparrow \infty, \\
& \left(\gamma^{-1} p+q\right)^{r}=p^{r} \gamma^{-r}+\sum_{k=1}^{\infty} \frac{1}{k !}\left(\prod_{j=0}^{k-1}(r-j)\right) p^{r-k} q^{k} \gamma^{k-r}
\end{aligned}
$$

The desired equation now follows by combining these asymptotic expansions.

Corollary A.1. Let $p$ and $q$ be as in Lemma A.1. Then, for $\gamma \downarrow 0$,

$$
\begin{aligned}
\Gamma\left(\mathrm{Ai}^{\prime}, \gamma^{-1} p+q\right)= & -\left(\pi^{-1 / 2} / 2\right)\left(\gamma^{-1} p\right)^{1 / 4} \exp \left(-(2 / 3)\left(\gamma^{-1} p\right)^{3 / 2}-q\left(\gamma^{-1} p\right)^{1 / 2}\right) \\
& \cdot\left[\left(1+\beta^{-1} \sqrt{p}\right)\left(1-\left(q^{2} / 4\right)\left(\gamma p^{-1}\right)^{1 / 2}\right)+(q / 4)\left(\left(q^{3} / 8-1\right)+\left(q^{3} / 8+3\right) \beta^{-1} \sqrt{p}\right) \gamma p^{-1}\right. \\
& \left.-(1 / 48)\left(-19+q^{3}+q^{6} / 8+\left(-7+7 q^{3}+q^{6} / 8\right) \beta^{-1} \sqrt{p}\right)\left(\gamma p^{-1}\right)^{3 / 2}\right], \\
\Gamma\left(\mathrm{Bi}^{\prime}, \gamma^{-1} p+q\right)= & \pi^{-1 / 2}\left(\gamma^{-1} p\right)^{1 / 4} \exp \left((2 / 3)\left(\gamma^{-1} p\right)^{3 / 2}+q\left(\gamma^{-1} p\right)^{1 / 2}\right) \\
& {\left[\left(1-\beta^{-1} \sqrt{p}\right)\left(1+\left(q^{2} / 4\right)\left(\gamma p^{-1}\right)^{1 / 2}\right)+(q / 4)\left(\left(q^{3} / 8-1\right)-\left(q^{3} / 8+3\right) \beta^{-1} \sqrt{p}\right) \gamma p^{-1}\right.} \\
& \left.+(1 / 48)\left(-19+q^{3}+q^{6} / 8-\left(-7+7 q^{3}+q^{6} / 8\right) \beta^{-1} \sqrt{p}\right)\left(\gamma p^{-1}\right)^{3 / 2}\right],
\end{aligned}
$$

where the remainders of $\mathcal{O}\left(\gamma^{2}\right)$ were omitted from within the square brackets.

Proof. Definition (4.2) and the identities $\operatorname{Ai}^{\prime \prime}(z)=z \operatorname{Ai}(z)$ and $\operatorname{Bi}^{\prime \prime}(z)=z \operatorname{Bi}(z)$ yield

$$
\begin{aligned}
\Gamma\left(\mathrm{Ai}^{\prime}, \gamma^{-1} p+q\right) & =\operatorname{Ai}^{\prime}\left(\gamma^{-1} p+q\right)-\beta^{-1} \sqrt{\gamma}\left(\gamma^{-1} p+q\right) \operatorname{Ai}\left(\gamma^{-1} p+q\right) . \\
\Gamma\left(\mathrm{Bi}^{\prime}, \gamma^{-1}+\bar{\chi}\right) & =\operatorname{Bi}^{\prime}\left(\gamma^{-1} p+q\right)-\beta^{-1} \sqrt{\gamma}\left(\gamma^{-1} p+q\right) \operatorname{Bi}\left(\gamma^{-1} p+q\right) .
\end{aligned}
$$

The desired result now follows from Lemma A.1. 
Lemma A.2. The function $\Gamma(\mathrm{Ai}, \bar{\chi})$ defined in (2.3) has no positive roots. For any $N \in \mathbf{N}$, there is $\varepsilon_{0}>0$ such that, for all $0<\varepsilon<\varepsilon_{0}, \Gamma(\mathrm{Ai}, \bar{\chi})$ has roots $A_{N, \sigma}^{\prime}<\ldots<A_{1, \sigma}^{\prime}<0$ satisfying

$$
\left|A_{n, \sigma}^{\prime}-\left(A_{n}+\beta^{-1} \sqrt{\gamma}\right)\right|<c_{a} \gamma, \text { for some } c_{a}>0 \text {. }
$$

Here, $A_{n}<0$ is the $n-$ th root of $\mathrm{Ai}$, see Fig. 2.1, and $\beta, \gamma$ are given in (4.1). For $\beta>1$ (equivalently, for $\left.0<\sigma<a^{2} / 4\right)$, the function $\Gamma\left(\mathrm{Bi}, \gamma^{-1}(1+\bar{\psi})\right)$ defined in (2.3) has a root $B_{0, \sigma}>0$ satisfying

$$
\left|B_{0, \sigma}-\left(\beta^{2}-1+2 \beta^{-1} \gamma^{3 / 2}\right)\right|<c_{b} \gamma^{3}, \text { for some } c_{b}>0
$$

Proof. The fact that there exist no positive roots of $\Gamma(\mathrm{Ai}, \bar{\chi})$ is immediate by the fact that $\operatorname{Ai}(\bar{\chi})>0$ and $\operatorname{Ai}^{\prime}(\bar{\chi})<0$ for all $\bar{\chi}>0$.

Next, the existence of $N$ discrete solutions may be proved in the following way. Let $\left|A_{N}\right|<X<$ $\left|A_{N+1}\right|$ be fixed and $I_{1}, \ldots, I_{N}$ be disjoint intervals around $A_{1}, \ldots, A_{N}$, respectively. It is easy to prove that the function $\Gamma(\mathrm{Ai}, \bar{\chi})$ is $\mathcal{O}(\sqrt{\gamma})$ close to Ai over $[-X, 0]$ in the norm introduced in $(4.10)$. Thus, for all $0<\gamma<\gamma_{0}$ (with $\gamma_{0}$ small enough), $\Gamma(\mathrm{Ai}, \bar{\chi})$ has $N$ distinct roots $A_{1, \sigma}^{\prime} \in I_{1}, \ldots, A_{N, \sigma}^{\prime} \in$ $I_{N}$ in $[-X, 0]$. The fact that these are ordered as $A_{N, \sigma}^{\prime}<\ldots<A_{1, \sigma}^{\prime}$ follows from $A_{N, \sigma}<\ldots<A_{1, \sigma}$ and the fact that the intervals $I_{1}, \ldots, I_{N}$ were chosen to be disjoint. The bounds on $A_{1, \sigma}^{\prime}, \ldots, A_{N, \sigma}^{\prime}$ may be derived by writing $A_{n, \sigma}^{\prime}=\sum_{\ell \geq 0} \varepsilon^{\ell / 6} a_{n, \sigma}^{(\ell)}$ and substituting into the equation $\Gamma(\mathrm{Ai}, \bar{\chi})=0$.

The existence of $B_{0, \sigma}>0$ and the bound on it may be established using Lemma A.1 (with $p=1+\bar{\psi}$ and $q=0)$.

Appendix B. Proof of Lemma 4.2 . Using definition (4.8), we calculate

$$
\mathcal{A}(\bar{\chi})-\Gamma(\mathrm{Ai}, \bar{\chi})=-\frac{\Gamma\left(\mathrm{Ai}, \gamma^{-1}+\bar{\chi}\right)}{\Gamma\left(\mathrm{Bi}, \gamma^{-1}+\bar{\chi}\right)} \Gamma(\mathrm{Bi}, \bar{\chi}) .
$$

To estimate the fraction in the right member, we apply standard theory for Airy functions $([2])$, see Appendix A. Using Lemma A.1 (with $p=1$ and $q=\bar{\chi}$ ), we find that

$$
\sup _{[X, 0]}\left|\exp \left(\frac{4}{3 \gamma^{3 / 2}}+\frac{2 \bar{\chi}}{\gamma^{1 / 2}}\right) \frac{\Gamma\left(\mathrm{Ai}, \gamma^{-1}+\bar{\chi}\right)}{\Gamma\left(\mathrm{Bi}, \gamma^{-1}+\bar{\chi}\right)}-\frac{1}{2} \frac{\beta+1}{\beta-1}\right|<c_{1} \sqrt{\gamma},
$$

for some $c_{1}>0$ and $\gamma$ small enough. Therefore,

$$
\sup _{[X, 0]}\left|\frac{\Gamma\left(\mathrm{Ai}, \gamma^{-1}+\bar{\chi}\right)}{\Gamma\left(\mathrm{Bi}, \gamma^{-1}+\bar{\chi}\right)}\right|<c_{2} \exp \left(-\frac{4+6 X \gamma}{3 \gamma^{3 / 2}}\right)
$$

for some $c_{2}>0$. Next, $\sup _{[X, 0]}|\Gamma(\mathrm{Bi}, \bar{\chi})| \leq c_{3}$, for some $c_{3}>0$, since $\mathrm{Bi}$ and $\mathrm{Bi}^{\prime}$ are uniformly bounded over $[X, 0]$. Combining these estimates, we find

$$
\sup _{[X, 0]}|\mathcal{A}(\bar{\chi})-\Gamma(\mathrm{Ai}, \bar{\chi})|<c_{4} \exp \left(-\frac{4+6 X \gamma}{3 \gamma^{3 / 2}}\right),
$$

for some $c_{4}>0$ and for all $\gamma$ small enough.

Next, differentiating (B.1), we calculate

$$
\begin{aligned}
\mathcal{A}^{\prime}(\bar{\chi})-\Gamma\left(\mathrm{Ai}^{\prime}, \bar{\chi}\right)= & \left(\frac{\Gamma\left(\mathrm{Ai}, \gamma^{-1}+\bar{\chi}\right) \Gamma\left(\mathrm{Bi}^{\prime}, \gamma^{-1}+\bar{\chi}\right)}{\left[\Gamma\left(\mathrm{Bi}, \gamma^{-1}+\bar{\chi}\right)\right]^{2}}-\frac{\Gamma\left(\mathrm{Ai}^{\prime}, \gamma^{-1}+\bar{\chi}\right)}{\Gamma\left(\mathrm{Bi}, \gamma^{-1}+\bar{\chi}\right)}\right) \Gamma(\mathrm{Bi}, \bar{\chi}) \\
& -\frac{\Gamma\left(\mathrm{Ai}, \gamma^{-1}+\bar{\chi}\right)}{\Gamma\left(\mathrm{Bi}, \gamma^{-1}+\bar{\chi}\right)} \Gamma\left(\mathrm{Bi}^{\prime}, \bar{\chi}\right) .
\end{aligned}
$$


Using Lemma A.1, we may bound the term in parentheses by

$$
\frac{c_{1}^{\prime}}{\sqrt{\gamma}} \exp \left(-\frac{4+6 X \gamma}{3}\left(\frac{1}{\gamma}\right)^{3 / 2}\right)
$$

for some $c_{1}^{\prime}>0$. Next, $\Gamma(\mathrm{Bi}, \bar{\chi})$ was uniformly bounded by a constant $c_{3}$ above. Also, the term $\Gamma\left(\mathrm{Bi}^{\prime}, \bar{\chi}\right)$ may be bound by a constant $c_{3}^{\prime}$, since

$$
\Gamma\left(\operatorname{Bi}^{\prime}, \bar{\chi}\right)=\operatorname{Bi}^{\prime}(\bar{\chi})-\beta \sqrt{\gamma} \operatorname{Bi}^{\prime \prime}(\bar{\chi})=\operatorname{Bi}^{\prime}(\bar{\chi})-\beta \sqrt{\gamma} \bar{\chi} \operatorname{Bi}(\bar{\chi})
$$

and the term multiplying it was bound in (B.2). These inequalities yield, then,

$$
\left\|\mathcal{A}^{\prime}(\bar{\chi})-\operatorname{Ai}^{\prime}(\bar{\chi})\right\|_{[X, 0]}<c_{2}^{\prime} \gamma^{-1 / 2} \exp \left(-\frac{4+6 X \gamma}{3 \gamma^{3 / 2}}\right)
$$

for some $c_{2}^{\prime}>0$ and for all $\gamma$ small enough. Equation (4.11) follows now from (B.3) and (B.4).

Appendix C. Proof of Lemma 4.3 . Definition (4.9) yields

$$
\mathcal{B}\left(\gamma^{-1} \bar{\psi}\right)-\Gamma\left(\mathrm{Bi}, \gamma^{-1}(1+\bar{\psi})\right)=-\frac{\Gamma\left(\mathrm{Bi}, \gamma^{-1} \bar{\psi}\right)}{\Gamma\left(\mathrm{Ai}, \gamma^{-1} \bar{\psi}\right)} \Gamma\left(\mathrm{Ai}, \gamma^{-1}(1+\bar{\psi})\right)
$$

To estimate the right member, we work as in Appendix B. Using Lemma A.1 twice (once with $p=\bar{\psi}, q=0$ and once with $p=1+\bar{\psi}, q=0)$, we obtain

(C.2) $\sup _{\left[\Psi_{R}, \Psi_{L}\right]}\left|E\left(\gamma^{-1}(1+\bar{\psi})\right) \frac{\Gamma\left(\mathrm{Bi}, \gamma^{-1} \bar{\psi}\right)}{\Gamma\left(\mathrm{Ai}, \gamma^{-1} \bar{\psi}\right)} \Gamma\left(\mathrm{Ai}, \gamma^{-1}(1+\bar{\psi})\right)\right|<c_{1} \gamma^{1 / 4}\left[\frac{E\left(\gamma^{-1}\left(1+\Psi_{L}\right)\right)}{E\left(\gamma^{-1} \Psi_{L}\right)}\right]^{2}$,

for some $c_{1}>0$ and $\gamma$ small enough.

Next, differentiating (C.1), we calculate

$$
\begin{aligned}
\mathcal{B}^{\prime}\left(\gamma^{-1} \bar{\psi}\right)-\Gamma^{\prime}\left(\mathrm{Bi}, \gamma^{-1}(1+\bar{\psi})\right)= & -\frac{\Gamma\left(\mathrm{Bi}, \gamma^{-1} \bar{\psi}\right)}{\Gamma\left(\mathrm{Ai}, \gamma^{-1} \bar{\psi}\right)} \Gamma\left(\mathrm{Ai}^{\prime}, \gamma^{-1}(1+\bar{\psi})\right) \\
& +\left(\frac{\Gamma\left(\mathrm{Bi}, \gamma^{-1} \bar{\psi}\right) \Gamma\left(\mathrm{Ai}^{\prime}, \gamma^{-1} \bar{\psi}\right)}{\left[\Gamma\left(\mathrm{Ai}, \gamma^{-1} \bar{\psi}\right)\right]^{2}}-\frac{\Gamma\left(\mathrm{Bi}^{\prime}, \gamma^{-1} \bar{\psi}\right)}{\Gamma\left(\mathrm{Ai}, \gamma^{-1} \bar{\psi}\right)}\right) \Gamma\left(\mathrm{Ai}, \gamma^{-1}(1+\bar{\psi})\right)
\end{aligned}
$$

Using Lemma A.1 and Corollary A.1 to estimate the right member, we find

(C.3) $\sup _{\left[\Psi_{R}, \Psi_{L}\right]}\left|E\left(\gamma^{-1}(1+\bar{\psi})\right)\left[\mathcal{B}^{\prime}\left(\gamma^{-1} \bar{\psi}\right)-\Gamma^{\prime}\left(\mathrm{Bi}, \gamma^{-1}(1+\bar{\psi})\right)\right]\right|<c_{1}^{\prime} \gamma^{-1 / 4}\left[\frac{E\left(\gamma^{-1}\left(1+\Psi_{L}\right)\right)}{E\left(\gamma^{-1} \Psi_{L}\right)}\right]^{2}$,

for some $c_{1}^{\prime}>0$ and $\gamma$ small enough.

The desired result follows from (C.2) and (C.3).

\section{REFERENCES}

[1] M. Abramowitz and I. A. Stegun (1965), Handbook of Mathematical Functions, Dover. 
[2] C. M. Bender and S. A. Orszag (1999), Advanced Mathematical Methods for Scientists and Engineers, Applied Mathematical Sciences 35, Springer-Verlag.

[3] P. N. Brown, G. D. Byrne, and A. C. Hindmarsh (1989), VODE: a variable-coefficient ODE solver, SIAM J. Sci. Statist. Comput. 10 1038-1051.

[4] E. A. Coddington and N. Levinson (1955), Theory of ordinary differential equations, McGraw-Hill, New York.

[5] U. Ebert, M. Arrayás, N. Temme, B. P. Sommeijer, and J. Huisman (2001), Critical conditions for phytoplankton blooms, Bull. Math. Biology 63(6) 1095-1124.

[6] P. G. Falkowski, R. T. Barber, and V. Smetacek (1998), Biogeochemical controls and feedbacks on ocean primary production, Science 281(5374) 200-206.

[7] K. Fennel and E. Boss (2003), Subsurface maxima of phytoplankton and chlorophyll: Steady-state solutions from a simple model, Limnol. Oceanogr. 48(4) 1521-1534.

[8] S. Ghosal and S. Mandre (2003), A simple model illustrating the role of turbulence on phytoplankton blooms, J. Math. Biology 46(4) 333-346.

[9] M. H. Holmes (1995), Introduction to Perturbation Methods, Texts in Applied Mathematics 20, Springer-Verlag.

[10] J. Huisman, P. van Oostveen, and F. J. Weissing (1999), Critical depth and critical turbulence: Two different mechanisms for the development of phytoplankton blooms, Limnol. Oceanogr. 44(7) 1781-1787.

[11] J. Huisman, N. N. Pham Thi, D. M. Karl, and B. P. Sommeijer (2006), Reduced mixing generates oscillations and chaos in the oceanic deep chlorophyll maximum, Nature $439322-325$.

[12] J. Huisman and B. P. Sommeijer (2002), Population dynamics of sinking phytoplankton in light-limited environments: Simulation techniques and critical parameters, J. Sea Research 48(2) 83-96.

[13] W. Hundsdorfer and J. G. Verwer (2003), Numerical Solution of Time-Dependent Advection-Diffusion-Reaction Equations, Series in Computational Mathematics 33, Springer-Verlag.

[14] H. Ishii and I. Takagi (1982), Global stability of stationary solutions to a nonlinear diffusion equation in phytoplankton dynamics, J. Math. Biology 16(1) 1-24.

[15] C. A. Klausmeier and E. Litchman (2001), Algal games: The vertical distribution of phytoplankton in poorly mixed water columns, Limnol. Oceanogr. 46(8) 1998-2007.

[16] K. H. Mann and J. R. N. Lazier (1996), Dynamics of Marine Ecosystems, Blackwell Science, Oxford.

[17] N. N. Pham Thi, J. Huisman, and B. P. Sommeijer (2005), Simulation of three-dimensional phytoplankton dynamics: competition in light-limited environments, J. Comput. Appl. Math. 174(1) 57-77.

[18] H. E. de Swart, H. M. Schuttelaars, and S. A. Talke (2007), Phytoplankton growth in turbid estuaries: a simple model, submitted to Continental Shelf Research.

19] I. Stakgold (1968), Boundary Value Problems of Mathematical Physics, vol.II, Macmillan, New York.

[20] K. Yoshiyama and H.Nakajima (2006), Catastrophic shifts in vertical distributions of phytoplankton. The existence of a bifurcation set, J. Math. Biology 52(2) 235-276. 